\title{
Hubble Space Telescope imaging of globular cluster candidates in low surface brightness dwarf galaxies $\star, \star \star$
}

\author{
M. E. Sharina ${ }^{1,2}$, T. H. Puzia ${ }^{3, \star \star \star}$, and D. I. Makarov ${ }^{1,2}$ \\ 1 Special Astrophysical Observatory, Russian Academy of Sciences, N. Arkhyz, KChR, 369167, Russia \\ 2 Isaac Newton Institute, Chile, SAO Branch \\ 3 Space Telescope Science Institute, 3700 San Martin Drive, Baltimore, MD21218, USA \\ e-mail: tpuzia@stsci.edu
}

Received 22 February 2005 / Accepted 26 May 2005

ABSTRACT

Fifty-seven nearby low surface brightness dwarf galaxies $\left(-10 \gtrsim M_{V} \gtrsim-16\right)$ were searched for globular cluster candidates (GCCs) using Hubble Space Telescope WFPC2 imaging in $V$ and $I$. The sample consists of 18 dwarf spheroidal (dSph), 36 irregular (dIrr), and 3 "transition" type (dIrr/dSph) galaxies with angular sizes less than $3.7 \mathrm{kpc}$ situated at distances $2-6 \mathrm{Mpc}$ in the field and in the nearby groups: $\mathrm{M} 81$, Centaurus A, Sculptor, Canes Venatici I cloud. We find that $\sim 50 \%$ of dSph, dIrr/dSph, and dIrr galaxies contain GCCs. The fraction of GCCs located near the center of dwarf spheroidal galaxies is $\gtrsim 2$ times higher than for dIrrs. The mean integral color of GCCs in dSphs, $(V-I)_{0}=1.04 \pm 0.16 \mathrm{mag}$, coincides with the corresponding value for Galactic globular clusters and is similar to the blue globular cluster sub-populations in massive early-type galaxies. The color distribution for GCCs in dIrrs shows a clear bimodality with peaks near $(V-I)_{0}=0.5$ and $1.0 \mathrm{mag}$. Blue GCCs are presumably young with ages $t \lesssim 1 \mathrm{Gyr}$, while the red GCC population is likely to be older. The detected GCCs have absolute visual magnitudes between $M_{V}=-10$ and -5 mag. We find indications for an excess population of faint GCCs with $M_{V} \gtrsim-6.5$ mag in both dSph and dIrr galaxies, reminiscent of excess populations of faint globular clusters in nearby Local Group spiral galaxies. The measurement of structural parameters using King-profile fitting reveals that most GCCs have structural parameters similar to extended outer halo globular clusters in the Milky Way and M 31, as well as the recently discovered population of "faint fuzzy" clusters in nearby lenticular galaxies.

Key words. galaxies: dwarf - galaxies: star clusters

\section{Introduction}

Low surface brightness dwarf galaxies $\left(M_{V}>-16\right.$, and central surface brightness $\mu_{\mathrm{V}} \gtrsim 22 \mathrm{mag} / \mathrm{arcsec}^{2}$ ) constitute the most numerous galaxy type in the local universe. Their formation mechanisms, their physical structure, and their contribution to the assembly of massive galaxies has attracted much attention for many years (e.g. Ferguson \& Binggeli 1994; Impey \& Bothun 1997; Bothun et al. 1997; Klypin et al. 1999; Kravtsov et al. 2004). Star formation in these low-mass stellar systems appears to be governed by a complex interplay of gravitational instabilities, turbulence, and gas thermodynamics (e.g. Elmegreen 2002; Pelupessy et al. 2004). In extreme cases, star formation culminates in the formation of massive star clusters that are likely to be the young counterparts of today's old

\footnotetext{
* Based on observations made with the NASA/ESA Hubble Space Telescope. The Space Telescope Science Institute is operated by the Association of Universities for Research in Astronomy, Inc. under NASA contract NAS 5-26555.

$\star \star$ Tables 1-3 are only available in electronic form at http://www . edpsciences.org

$\star \star \star$ ESA Fellow, Space Telescope Division of ESA.
}

globular clusters (e.g. Larsen \& Richtler 2000). This mode of star-formation is observed in numerous nearby dwarf irregular galaxies (dIrr; e.g. Billett et al. 2002; Hunter \& Elmegreen 2004), but appears to have ceased a long time ago in dwarf spheroidal (dSph) and dwarf elliptical galaxies (dE; see Lotz et al. 2004) $)^{1}$. While $\mathrm{dE}$ and $\mathrm{dSph}$ galaxies are predominantly found in the vicinity of massive galaxies in galaxy groups and rich galaxy clusters, dIrr galaxies are predominantly located in the field and in loose groups. The conditions for globular cluster formation in dwarf galaxies at a given galaxy mass might, therefore, be a sensitive function of environmental density. It is still not clear whether $\mathrm{dE}, \mathrm{dSph}$, and dIrr share similar formation and/or early evolution histories (Davies \& Phillipps 1988; Marlowe et al. 1999). However, the three galaxy types share one property: they all harbor globular clusters that are older than several Gyr, which indicates that at least early globular cluster formation took place irrespective of the morphological type. One can use these globular clusters to investigate the early star formation and chemical evolution histories of these

\footnotetext{
1 We explicitly consider in our sample the type of tidal dwarf galaxies (e.g. Weilbacher 2002).
} 
galaxies. Because they are composed of stars of one age and chemical composition, globular clusters offer a unique tool to access the star formation histories of individual galaxies (e.g. Ashman \& Zepf 1998; Kissler-Patig 2000; Harris 2001).

In the present epoch, the formation of massive bound star clusters seems to be associated with high-pressure environment and powerful star formation events (e.g. Elmegreen \& Efremov 1997; Ashman \& Zepf 2001). A high-pressure environment naturally occurs in dwarf galaxies due to their low metallicities and high critical densities for star formation. Since globular clusters sample the chemical conditions during major star formation events in a galaxy, the chemical composition of globular clusters in $\mathrm{dE}, \mathrm{dSph}$, and dIrr galaxies might provide crucial information on star formation histories and mechanisms that offer important input for hierarchical galaxy formation models. We, therefore, embarked on a spectroscopic survey of globular clusters in nearby low surface brightness galaxies in and outside the Local Group (LG).

In this paper, we present the photometric study of globular cluster candidates. In Sect. 2 we describe the galaxy sample and data reduction steps. Section 3 deals with the cluster candidate selection, while in Sect. 4 we investigate the properties of the globular cluster candidates (GCCs).

\section{Observations and data reduction}

Before discussing the properties of globular cluster candidates in nearby dwarf galaxies, we briefly summarize the main characteristics of the objects of interest. Globular clusters are centrally concentrated, mostly spherical systems with masses of $10^{4} \leq M_{\odot} \leq 10^{6.6}$, luminosities from $M_{V}=-10.55$ (Mayall II in M 31) to +0.2 mag (AM-4 in the Milky Way), and halflight radii ranging from $\sim 0.3$ to $\sim 25 \mathrm{pc}$. They are bound objects whose lifetimes may exceed a Hubble time. So far, globular clusters have been detected in 12 of the 36 Local Group galaxies (Hodge et al. 2002). Table 1 presents a list of galaxies that were searched for globular cluster candidates in this study.

Numerical values in the Cols. 3, 4 and 6-8 were extracted and/or computed from data presented in Karachentsev et al. (2004). Surveying Table 1 shows that our sample is composed of 18 dwarf spheroidal (dSph: $T<-1$ ), 36 dwarf irregular (dIrr: $T>9$ ), and 3 intermediate-type (dSph/dIrr: $T=-1$ ) galaxies with mean surface brightnesses $\mu_{B}>23 \mathrm{mag} / \mathrm{arcsec}^{2}$ and angular sizes less than $3.7 \mathrm{kpc}$. All galaxies, except KK $84^{2}$, are situated at distances $\sim 2-6 \mathrm{Mpc}$ in the field and in the nearby groups: M 81, Centaurus A, Sculptor, Canes Venatici I cloud (see Table 2 for details). We find globular cluster candidates in 10 of $18 \mathrm{dSphs}, 18$ of $36 \mathrm{dIrrs}$, and 2 of 3 intermediatetype dwarfs. In general, roughly $50 \%$ of all surveyed galaxies contain globular cluster candidates, irrespective of morphological type.

The galaxies were surveyed with the HST Wide Field and Planetary Camera 2 (WFPC2; snapshot programs GO-8192, GO-8601) with 600-second exposures taken in the F606W and F814W filters for each object. Accurate distances to 111 nearby galaxies were determined in these snapshot programs (e.g.

\footnotetext{
${ }^{2} \mathrm{KK} 84$ is located at a distance of $9.7 \mathrm{Mpc}$.
}

Karachentsev et al. 2003) and provide us an unique benchmark to study properties of globular cluster systems in a number of low surface brightness dwarf galaxies. Karachentsev et al. (2000a) searched for globular cluster candidates in dSph galaxies of the M 81 group. We extended this work using our selection criteria and also included other low-mass galaxies in this sample. In general, surveying the full area of all our dwarf galaxies, given their relatively small angular sizes of $\lesssim 2^{\prime}$, allows us to study the spatial distribution of globular cluster candidates.

In the following we briefly discuss a few particularly interesting galaxies. WFPC2 images with marked GCCs in UGC 3755 and Holmberg IX are shown in Figs. 1a,b. These two galaxies, which have different properties and are located in different environments (see Table 2), have the largest numbers of globular cluster candidates among our sample galaxies. UGC 3755 is an isolated dwarf irregular galaxy (Karachentsev et al. 2004). Holmberg IX is a tidal dwarf companion of M 81 (Yun et al. 1994; Boyce et al. 2001). We searched for globular cluster candidates in other tidal dwarf companions of M 81, namely BK3N, Arp-loop (A0952+69) and Garland, but found no GCCs in these galaxies. A faint GCC $\left(M_{V, 0}=-5.2\right)$ in BK3N is located outside the boundary of the galaxy and probably belongs to M 81. Six dSph galaxies contain GCCs located near their centers. KK84 and KK221 have the largest number of GCCs among our dSph sample (see Table 2 and Figs. $1 \mathrm{c}$,d). Overplotted in Fig. 1d is the isophote of constant surface brightness $\mu_{B} \sim 26.5 \mathrm{mag} / \mathrm{arcsec}^{2}$. All globular cluster candidates in KK221 are located within the boundaries of this isophote, but the location of the brightest cluster and the whole globular cluster system seems to be shifted from the central position in the galaxy. We can only speculate whether KK221 has an elongated orbit and experienced strong tidal forces from NGC 5128, which moves its globular cluster system from a centered position.

Globular cluster candidates were selected using the FIND task of the DAOPHOT-II (Stetson 1987) package implemented in MIDAS. The detection threshold was set at 4- $\sigma$ above the background. The minimum full width at half maximum input parameter $(F W H M)$ used was $\sim 0.2^{\prime \prime}$ (The stellar FWHM is $\sim 0.15^{\prime \prime}$ ). Photometry was performed using the PHOT task of DAOPHOT-II with a 12 pixel/1.2" radius. To convert instrumental magnitudes $\mathrm{F} 606 \mathrm{~W}$ and $\mathrm{F} 814 \mathrm{~W}$ into the standard Johnson-Cousins system, we used the surface photometry recipes and equations presented by Holtzman et al. (1995). Surface brightness profiles and growth curves were computed from the aperture photometry results. The growth curves were extrapolated to an infinitely large aperture. Finally, the magnitudes of GCCs were corrected for Galactic extinction using reddening maps from Schlegel et al. (1998), and absolute magnitudes were computed by applying the distance moduli reviewed in Karachentsev et al. (2004). Half-light radii and linear projected separations of GCCs from the centers of parent galaxies were converted to a linear measure in parsecs.

We performed photometry on 37 dwarf galaxies using the WFPC2 images and the surface photometry recipes and equations given in Holtzman et al. (1995). All steps were identical to those used in Makarova (1999), and we refer the reader to 

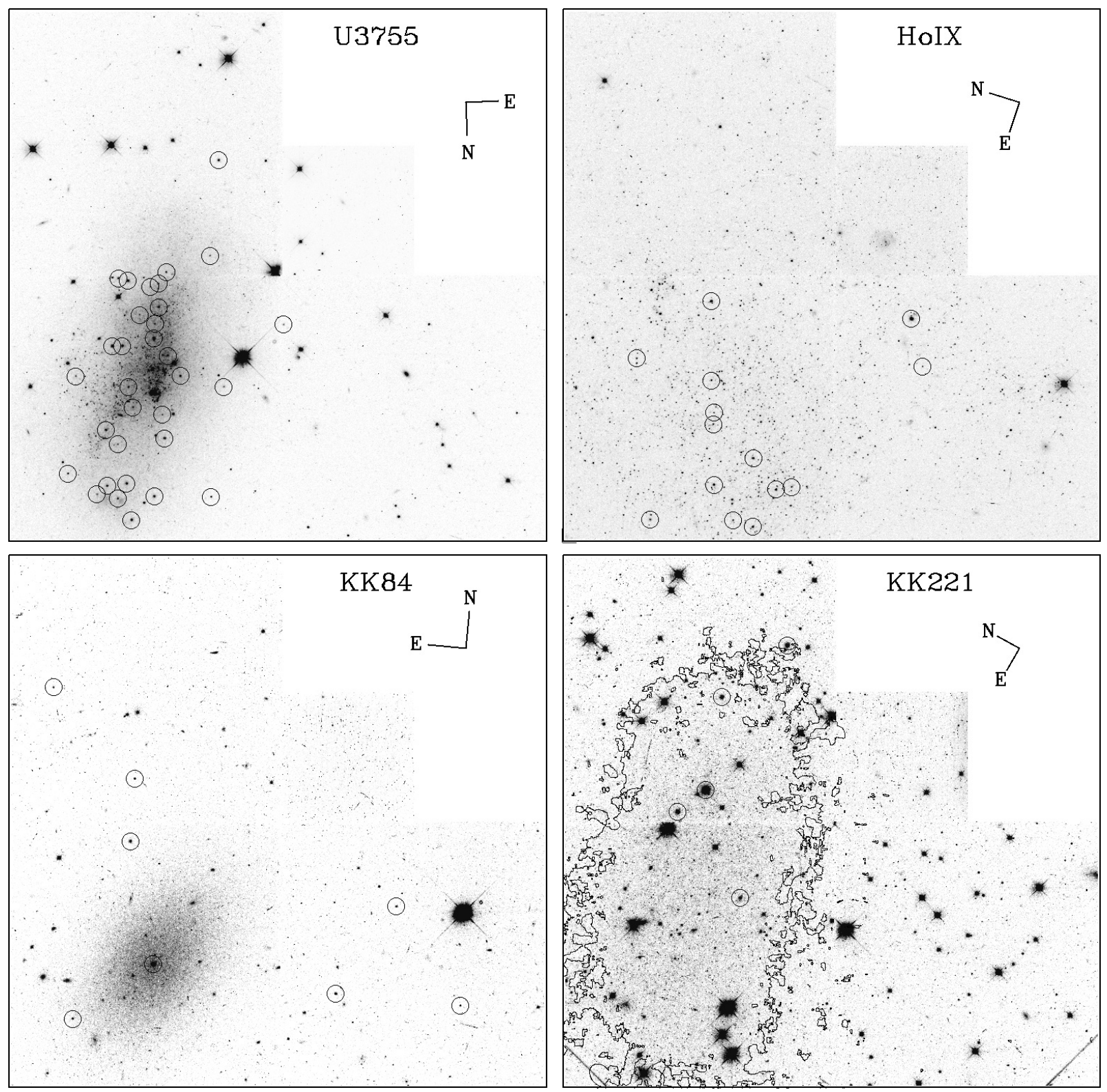

Fig. 1. HST/WFPC2 images for U3755, Holmberg IX, KK84, and KK221 which host the richest GCC systems in our sample in their morphology classes (see Sect. 1 for details).

this work for further details. Integrated absolute $V$ magnitudes are listed in Table 1.

\section{Cluster candidate selection}

Our primary target lists include stars, galaxies, and star clusters. In order to select globular cluster candidates, we applied a color selection cut of $0.3<(V-I)_{0}<1.5$, which is the full range expected for clusters older than $100 \mathrm{Myr}$ and metallicities $-2.5<\left[Z / Z_{\odot}\right]<0.5$ (e.g. Bruzual \& Charlot 2003). We selected round objects $(F W H M(x) \simeq F W H M(y))$ with halflight radii of $2<F W H M<9$ pix. Reduced to linear measure in parsecs using the distance measurements of Karachentsev et al. (2004), this range corresponds to projected half-light radii $3<r_{\mathrm{h}}<20 \mathrm{pc}$, which are within values typical for Galactic globular clusters (Harris 1996, and 2003 update). Then we performed a visual inspection of all selected GCCs on the WFPC2 images. The high angular resolution of HST helped us to reject objects which showed evidence of spiral or disturbed substructure (most likely background galaxies) from the list of globular cluster candidates.

We applied a lower absolute magnitude limit for our GCCs of $M_{V}=-5.0 \mathrm{mag}$. For all our images, this magnitude limit is much brighter than the photometric limit of the images $(V \sim$ $25.0 \mathrm{mag}$ ), hence no completeness corrections are necessary. In other words, our sample is complete down to $\sim 2.5 \mathrm{mag}$ past the presumed turnover of the globular cluster luminosity function (e.g. Harris 2001). Figure 2 shows that GCCs detections for the galaxy KK84 located at a distance of $9.7 \mathrm{Mpc}$ is complete down to $M_{V, 0} \approx-6.6$. Assuming that globular clusters in this galaxy have an intrinsic luminosity function similar to the Milky Way 


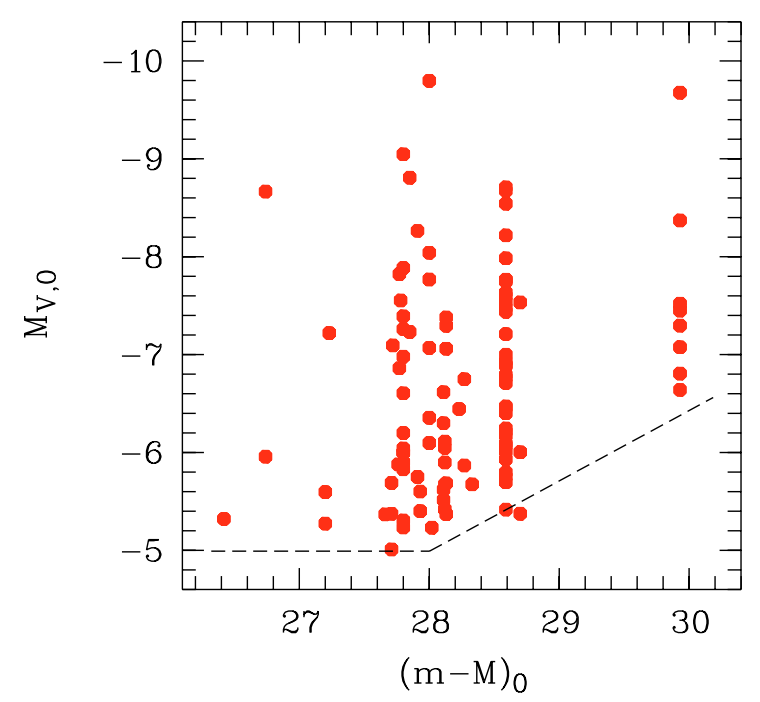

Fig. 2. Distance modulus versus absolute $V$ magnitude for GCCs, identified in this study and listed in Table 2. Our sample is complete down to $M_{V} \approx-5$ for all our sample galaxies, except KK84. Detections of GCCs in KK84 are complete down to $M_{V} \approx-6.6 \mathrm{mag}$.

globular cluster luminosity function, we detected $\sim 80 \%$ of all globular clusters.

Finally, we fit surface brightness profiles to our GCCs with the King law (King 1962)

$\mu_{i}=k\left(\frac{1}{\sqrt{1+\left(r / r_{\mathrm{c}}\right)^{2}}}-\frac{1}{\sqrt{1+c^{2}}}\right)^{2}$.

We minimized the $\chi^{2}$ function:

$\chi^{2}=\sum_{i} \frac{\left(\mu_{i}-\bar{\mu}_{i}\right)^{2}}{\sigma_{i}^{2}}$,

where $\mu_{i}$ is an average surface brightness inside a circular ring aperture, $\bar{\mu}_{i}$ the predicted value for the same circular ring, and $\sigma_{i}$ the corresponding photometric error. Nonlinear least-square fits give us the following parameters: $r_{\mathrm{c}}-$ core radius, $r_{\mathrm{t}}-$ truncation radius of the King model, concentration parameter $c=r_{\mathrm{t}} / r_{\mathrm{c}}$, and $\mu_{0}-$ central surface brightness of the GCC. The King law approximation provides an additional argument for rejecting background galaxies from our GCCs list. King (1962) emphasized that "relative to globular clusters, giant elliptical galaxies have an excess of brightness near the center". Hence, we rejected objects with a central excess brightness and/or uncertain output parameters. About $10 \%$ of the sample was removed in this way by visual inspection. Most of the rejected sources have $V-I>1.4$ and are, therefore, likely to be background galaxies.

The final list of all GCCs in our sample galaxies is presented in Table 2. Parameters obtained by the King-law approximation of GCC surface brightness profiles are listed in Table 3.

It should be mentioned that, even after applying all our selection procedures, we cannot be certain that all objects in our list are genuine globular clusters. Elliptical galaxies at intermediate redshifts are hard to distinguish from globular clusters using only $V$ and $I$ magnitudes. Unresolved starbursts with

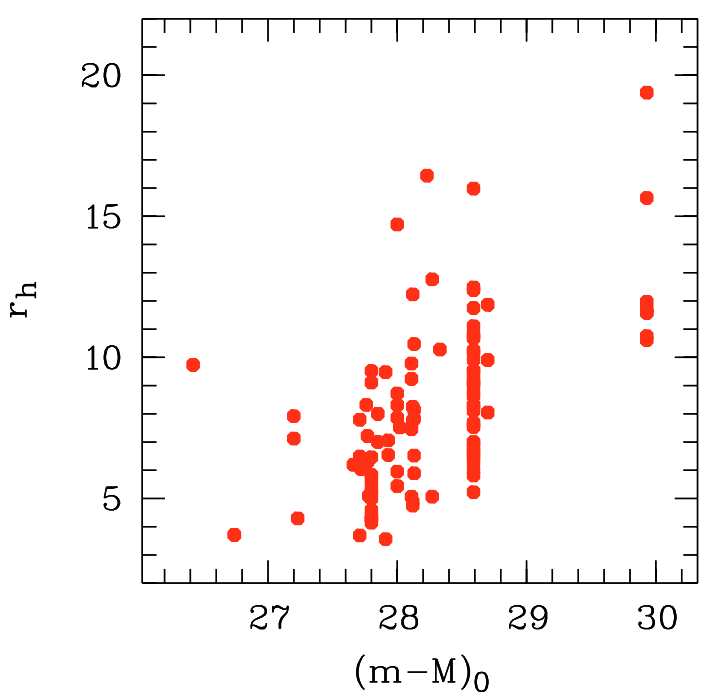

Fig. 3. Half-light radius of globular cluster candidates (see Table 3) versus distance modulus of their host galaxies. Our sample is composed mainly of GCCs with core radii $\sim 3-13$ pc (see Sect. 3 for details).

ages $\leq 300$ Myr at redshifts $z \sim 0.1-1.0$ are potential contaminants (Puzia et al. 2004). We estimated the number of background galaxies down to $I=22.5 \mathrm{mag}$ based on FORS Deep Field data (Heidt et al. 2003). We expect $\sim 3-4$ background galaxies within the WFPC2 field of view with colors resembling those of globular clusters. Eleven of our sample GCCs are located outside the $\mu_{B} \sim 26.5 \mathrm{mag} / \mathrm{arcsec}^{2}$ isophotes of their respective host galaxies. The probability that they are background galaxies is higher than for the other GCCs. Five of these GCCs are located in dIrr and dSph/dIrr galaxies and have colors $(V-I)_{0}>1.2$ and absolute magnitudes within the range $-5.3<M_{V_{0}}<-6.3$. Another six GCCs are located outside the $\mu_{B} \sim 26.5 \mathrm{mag} / \operatorname{arcsec}^{2}$ isophote, which have $0.6<(V-I)_{0}<1$ and $-5.2<M_{V, 0}<-6.6$. Four of these belong to dSphs. Hence, we estimate the contamination of background objects within the boundaries of the $\mu_{B} \sim 26.5 \mathrm{mag} / \mathrm{arcsec}^{2}$ isophote for our galaxies with $(V-I)_{0}<1.2$ to be $\lesssim 10 \%$.

Before analyzing properties of GCCs we investigated our sample for observational selection effects. Figure 3 shows the half-light radii of our GCCs (see Table 3) as a function of the distance modulus of their host galaxy. Our sample is mainly composed of GCCs with core radii $\sim 3-13 \mathrm{pc}$. With the current dataset, we cannot rule out the presence of GCCs fainter than $M_{V} \approx-6.5$ and with core radii $\lesssim 10 \mathrm{pc}$ in the $\mathrm{dSph}$ galaxy KK84, which is the most distant host galaxy in our sample, situated at $9.7 \mathrm{Mpc}$.

On the other hand, we detected five GCCs with $r_{\mathrm{c}}>12 \mathrm{pc}$ in galaxies that are more distant than $3.8 \mathrm{Mpc}$. This fact might be caused by two reasons. Firstly, the size of space volume projected onto an image pixel increases with increase in the distance to galaxies. Errors of structural parameters grow accordingly. However, we do not find a significant increase in the mean error for these five objects compared to the rest of our sample. Secondly, the surveyed area of galaxies grows with distance. This might lead to a higher detection rate of large GCCs in the outskirts of these galaxies. Two of these five objects are 
located at the largest galactocentric radii with respect to their host galaxy (see Sect. 5 for details). This in turn means that we might miss a few extended globular cluster candidates in nearby galaxies at large galactocentric radii. Larger field coverage for nearby systems would help to resolve this issue. For the remainder of this work, we keep in mind that the lack of extended GCCs in nearby galaxies is a potential bias of our current dataset.

\section{Properties of globular cluster candidates}

\subsection{Colors}

Figure 4 shows a color-magnitude diagram for all GCCs. Colors and magnitudes were corrected for Galactic foreground extinction using the reddening maps of Schlegel et al. (1998). We have no means to correct for internal reddening of the observed dIrr galaxies. However, given the similarity of these systems to nearby dIrr galaxies we estimated that this correction is $E_{(B-V)} \lesssim 0.1 \mathrm{mag}$ (James et al. 2005). Hence, we refer in the following to foreground extinction corrected magnitudes and colors by indexing them with a zero.

A KMM test (Ashman et al. 1994) for GCCs in dIrrs returns peaks at $(V-I)_{0}=0.48 \pm 0.02$ and $1.02 \pm 0.03 \mathrm{mag}$ with dispersions 0.12 and $0.22 \mathrm{mag}$ for the blue and red peak, respectively. The peak of the $\mathrm{dSph}$ distribution is located at $(V-I)_{0}=1.01 \pm 0.03 \mathrm{mag}$ and has a dispersion of $0.18 \mathrm{mag}$. The red peak GCC sub-population in dIrrs and most GCCs in dSph galaxies cover the same $(V-I)_{0}$ color range as the ancient Galactic and M 31 globular clusters (see Fig. 1 in Puzia et al. 2004 ). More than $\sim 50 \%$ of all globular cluster systems in massive early-type galaxies show indications for multi-modality, with mean peak colors at $(V-I)_{0} \approx 0.95$ and $\sim 1.18$ mag (e.g. Kundu \& Whitmore 2001a,b; Larsen et al. 2001). So far most of these globular cluster systems have been found to be old (Puzia et al. 1999; Jordán et al. 2002). A comparison of $(V-I)_{0}$ colors reveals that most GCCs in dSphs and the red-peak GCCs in dIrrs are similar to blue globular clusters in early-type galaxies (see vertical lines in Fig. 4), which implies similar ages and metallicities. The blue sub-population of GCCs in dIrr galaxies is significantly bluer and suggests much younger ages and/or lower metallicities.

Figure 5 shows the color histogram for GCCs in dIrr and dSph galaxies. The distribution of $(V-I)_{0}$ colors exhibits an obvious bimodality for GCCs in dIrr galaxies, in contrast to the distribution of GCCs in dSph galaxies, which shows a single mode distribution. The clear difference between the two distributions is underlined by a non-parametric probability density estimate (Silverman 1986). The color of the red peak in the dIrr distribution is virtually identical with the mean of the $\mathrm{dSph}$ distribution. Their dispersions are also very similar. This points to the fact that both galaxy types host similar globular cluster populations.

We compare the color distributions of GCCs in dIrr and dSphs with that of $\mathrm{dE}$ galaxies. In the bottom panel of Fig. 5 we present the color histogram of globular cluster candidates in 69 dwarf elliptical galaxies in the Virgo and Fornax galaxy clusters and the Leo group, using data taken from

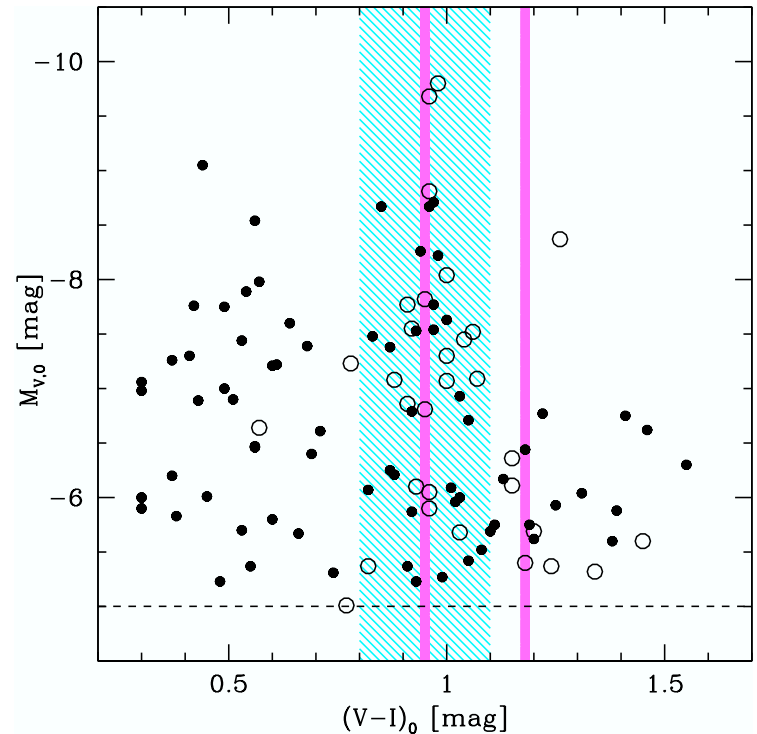

Fig. 4. Color-magnitude diagram of globular cluster candidates in $\mathrm{dSph}$ (open circles) and dIrr galaxies (solid dots), identified in this study and listed in Table 2. The dashed line indicates the limit of our photometry (see Sect. 3 for details). Note that there are virtually no GCCs in dSph galaxies with colors bluer than $(V-I)_{0} \approx 0.7 \mathrm{mag}$. The hatched region shows the color range where most Galactic and M 31 globular clusters are found (see Puzia et al. 2004). The two vertical lines indicate colors of blue and red sub-populations in massive earlytype galaxies (e.g. Kundu \& Whitmore 2001a,b; Larsen et al. 2001).

Lotz et al. (2004). The peak of this distribution is at $(V-I)_{0}=$ $0.90 \pm 0.03 \mathrm{mag}$. The $\mathrm{dE}$ color distribution, in particular the red end, is similar to the one of dSph GCCs and the red peak of the dIrr distribution. It is remarkable that the $\mathrm{dE}$ color distribution is shifted to bluer colors by $\sim 0.1 \mathrm{mag}$ with respect to the mean color of the $\mathrm{dSph}$ and the red peak of the dIrr GCC sub-population. This indicates a mean difference in age and/or metallicity. To better assess the reality of this color difference, we carried out an independent photometry of GCCs in one galaxy (VCC1254) from the sample of Lotz et al. (2004). For this purpose we applied our photometric routines to the identical images as used in the Lotz et al. study and compared our results to their work. We found a small systematic offset $\Delta(V-I)=0.04 \pm 0.05$ for 21 common objects, in the sense that our values tend to be redder. This indicates that different photometric approaches might have a small influence on our conclusions. A Kolmogorov-Smirnov test shows that there is a $0.03 \%$ likelihood that the $\mathrm{dE}$ and $\mathrm{dSph}$ color distributions were drawn from the same sample. This likelihood decreases significantly for the other combinations dE-dIrr and dSph-dIrr.

SSP models (e.g. Bruzual \& Charlot 2003) predict that for a solar-metallicity, $13 \mathrm{Gyr}$ old stellar population a $\Delta(V-I)_{0}=0.1$ difference translates into $\sim 7 \mathrm{Gyr}$ younger ages (at the same metallicity) and/or a $\sim 0.6$ dex smaller metallicity (at the same age). Because of the age-metallicity degeneracy of photometric colors, it is difficult to derive individual ages and metallicities from only two colors. However, comparison of the colors with current SSP models shows that $(V-I) \approx 1.0$ mag is consistent with stellar populations with a certain combination of ages older than $1 \mathrm{Gyr}$ and metallicities $[\mathrm{Z} / \mathrm{H}] \gtrsim-1.4 \mathrm{dex}$. 


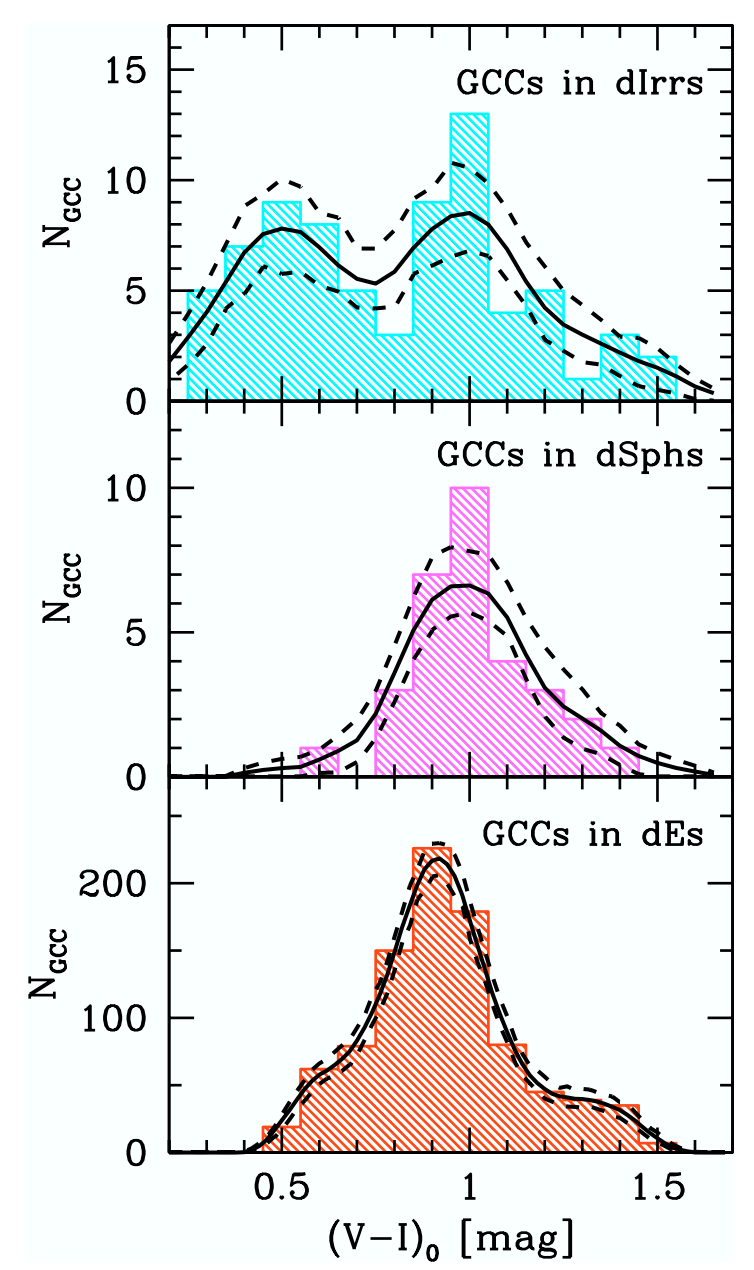

Fig. 5. Color distributions of globular cluster candidates in dSph, dIrr, and $\mathrm{dE}$ galaxies. The upper and middle panel show our data, while the bottom histogram was constructed from data taken from Lotz et al. (2004). Solid lines indicate a non-parametric probability density estimate using an Epanechnikov kernel (Silverman 1986). Dashed lines show $90 \%$ confidence limits.

The blue peak of the dIrr color distribution, on the other hand, is consistent with stellar populations that have ages $\lesssim 1 \mathrm{Gyr}$ and metallicities $[\mathrm{Z} / \mathrm{H}] \gtrsim-2.0$ dex. Thus, we suggest that red-peak GCCs in dIrr and most GCCs in dSph are metal-rich globular clusters with intermediate to old ages. Blue GCCs in dIrr galaxies are likely to be relatively young globular clusters, probably similar to populous star clusters found in several star-forming nearby galaxies (e.g. Larsen \& Richtler 2000).

\subsection{Luminosity function}

In addition to a blue and most likely younger population of GCCs in dIrrs, the color distributions revealed a red and presumably ancient population in both $\mathrm{dSph}$ and dIrr galaxies. To investigate the luminosity functions (LFs) of these subpopulations individually, we split the dIrr sample at $(V-I)_{0}=$ 0.75 mag (see Fig. 5) into red and blue cluster candidates. The corresponding LFs are shown in Fig. 6 down to $\sim 2.5$ mag past the turnover of a typical globular cluster luminosity function, which is indicated by a vertical line (Harris 2001).

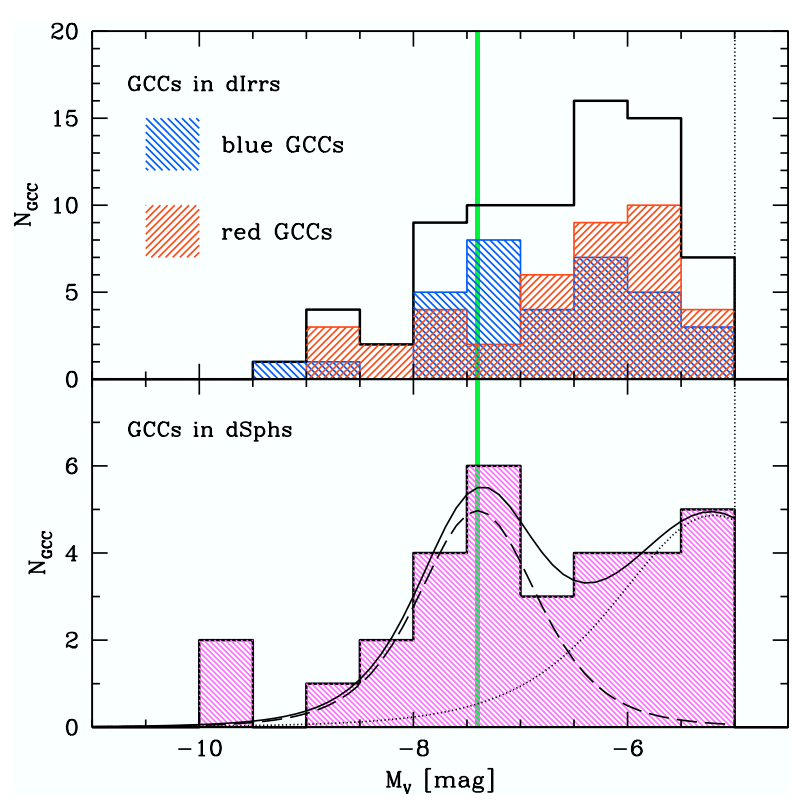

Fig. 6. Luminosity functions of globular cluster candidates in dIrr (top panel) and dSph galaxies (bottom panel). The luminosity functions of red and blue GCCs in dIrr galaxies are also shown as hatched histograms. The shaded vertical line indicates the turnover of the Galactic globular cluster luminosity function. The dotted line represents our photometric limit.

The LF of blue GCCs seems broad with a turnover somewhere between $M_{V} \approx-7.5$ and $-6.0 \mathrm{mag}$. The fact that we see a turnover for these supposedly young clusters is remarkable, since constant power-law slopes down to faint magnitudes are observed in other globular cluster systems of similar age (e.g. Whitmore et al. 1999; Goudfrooij et al. 2004). While these clusters are found in dense environments of ongoing mergers and massive early-type galaxies, our sample GCCs are located in low-mass galaxies in loose groups and in the field. The reason that we see a turnover in a young cluster system might be a consequence of fundamentally different mechanisms of star cluster formation. The star formation rate in our sample dIrrs is relatively low. Hence, slow spontaneous instabilities are likely to dominate the star formation process but do not support the formation of gravitationally bound low-mass star clusters. Another possible explanation might be more efficient destruction processes, such as infant mortality (Lada \& Lada 2003), which is thought to be the consequence of early gas ejection. In general, since physical differences between young star clusters are related to the pressure differences of the environment in which they form (e.g. Elmegreen \& Efremov 1997; Ashman \& Zepf 2001), slight differences in luminosity functions of young globular cluster systems in different environments cannot be excluded.

The LF of red GCCs in dIrrs turns over at a much fainter magnitude $M_{V} \approx-6$ mag than for their blue counterparts. We can only speculate whether this is due to a difference in formation mechanisms and ages/metallicities, or simply due to contamination by background galaxies. We point out that contamination by stellar crowding is more likely to occur in dIrr galaxies, which are populated by bright stars. However, stellar 
crowding is unlikely to explain the excess of objects, which is also found in dSph galaxies (see below).

For GCCs in dSphs the luminosity function shows a turnover at around $M_{V}=-7.4$ mag. Similar turnover magnitudes are also found in many other globular cluster systems (Harris 2001). In addition to a roughly log-normal LF for bright objects, we found that the luminosity functions of GCCs fainter than $M_{V}=-6.5 \mathrm{mag}$ are steadily increasing towards fainter magnitudes, possibly resembling a dynamically less evolved population of clusters (Gnedin \& Ostriker 1997; Fall \& Zhang 2001). The existence of an excess population of faint globular cluster candidates in dSphs is surprising and is reported on here for the first time. A scaled Student $t_{5}$-distribution

$t_{5}\left(m \mid M_{V, 0}, \sigma_{t}\right)=\frac{8}{3 \pi \sigma_{t} \sqrt{5}}\left(1+\frac{\left(m-M_{V, 0}\right)^{2}}{5 \sigma_{t}^{2}}\right)^{-3}$,

which is the best approximation to the observed Galactic globular cluster luminosity function (Secker 1992, see dashed line in the bottom panel of Fig. 6), shows good agreement with the bright peak ${ }^{3}$, where $M_{V, 0}=-7.4$ and $\sigma_{t}=0.6$. The faint cluster excess can be approximated well by a second $t_{5}$-peak with a mean at $M_{V, 0} \approx-5.2 \mathrm{mag}$ and roughly twice as broad a dispersion as the bright peak. This is reminiscent of the composite luminosity function of globular and open clusters in the Milky Way. However, since this is a composite luminosity function for GCCs in different galaxies, various dynamical processes are lumped together in one sample. This and the possibility of variable internal reddening might account for at least part of the spread in the observed luminosity function.

We note that a similar excess population of faint star clusters has been discovered in the low-mass spiral M 33 (Chandar et al. 2001) and in nearby lenticular galaxies (Brodie \& Larsen 2002). However, the nature of these faint excess clusters is difficult to assess based on two-color photometry.

For KK84 and UGC 3755, which host the richest GCC systems in their morphology class, we investigate their GCC luminosity distributions individually. The GCC luminosity function of the dSph galaxy KK84 peaks near $M_{V} \approx-7.3 \mathrm{mag}$. For the dIrr galaxy UGC 3755 we find peaks near $M_{V} \approx-7.0 \mathrm{mag}$ for both the blue and red sub-population. Both galaxies are relatively massive compared to the rest of the sample and show typical turnover magnitudes.

In summary, given the number statistics of our data, we can say that, compared to a typical globular cluster luminosity function, both the dSph and dIrr GCC populations exhibit an excess of faint globular cluster candidates. Whether this is due to a genuine new population of low-mass star clusters or due to contamination by marginally resolved background galaxies will be resolved with spectroscopic data.

\subsection{Spatial distributions}

In the following, we compare the composite spatial distribution of GCCs in dSph and dIrr galaxies. We divide the population of GCCs in dIrr galaxies into red and blue objects at

\footnotetext{
3 This approximation neglects two very bright GCCs with magnitudes $M_{V} \approx-10$.
}

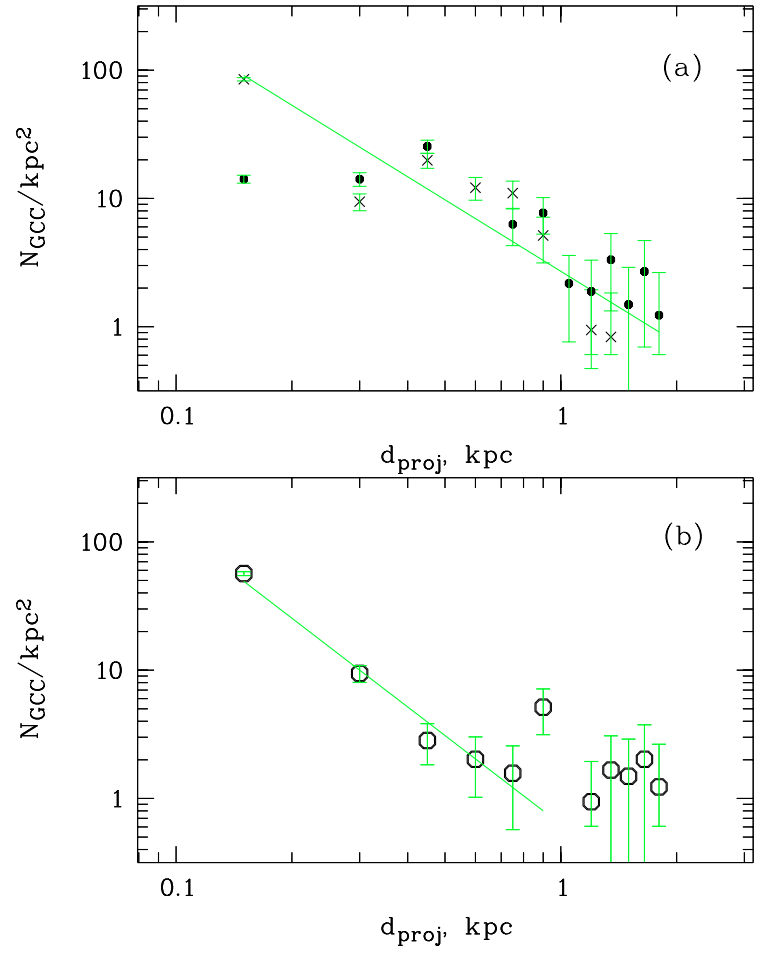

Fig. 7. Radial distribution of GCCs in dIrr galaxies (panel a) and dSph galaxies (panel b). The sample of GCCs in dIrrs is divided into blue GCCs with $(V-I)_{0}<0.75$ (dots) and red GCCs $(V-I)_{0}>0.75$ (crosses). Plotted here is the logarithm of the surface density of GCCs per square kpc (evaluated in $0.15-\mathrm{kpc}$ bins) vs. the logarithmic projected distance from the galaxy center, in kpc.

$(V-I)_{0}=0.75$ mag. In Fig. 7 we plot the logarithmic number density of GCCs per square kpc versus the logarithm of the linear projected separation from the galaxy center in kpc for dIrr and dSph galaxies. Radial distributions of blue and red GCCs in dIrrs are shown by different symbols and reveal somewhat different slopes. A power law for the surface density of the form $\rho=r^{-x}$ gives a good fit to our data. We find that the surface density profiles of globular cluster systems in dIrr galaxies are "flatter", $\left(x_{\text {red }} \approx 1.1\right)$ and $\left(x_{\text {blue }} \approx 1.85\right)$, than those in $\mathrm{dSph}$ galaxies $(x \approx 2)$. The profile for GCCs in $\mathrm{dSph}$ galaxies seems to flatten out beyond $\sim 1 \mathrm{kpc}$ galactocentric distance. Hence, the fit includes only the inner part of the GCC population. The profiles of the GCC population in dIrr galaxies do not show a flattening at large galactocentric distances.

These values are in good agreement with those found for globular cluster systems in elliptical ( $x=0.8-2.6$, Puzia et al. 2004) and dwarf elliptical galaxies ( $x=1.6 \pm 0.4$, Durrell et al. 1996). Moreover, Harris (1986) found $x=3.5 \pm 0.5$ for the combined spatial distribution of globular clusters in NGC 147, NGC 185, and NGC 205. Minniti et al. (1996) constructed a composite dwarf elliptical galaxy from all early-type dwarf galaxies in the Local Group and computed $x=2.1$.

\subsection{Structural parameters}

Under the superb spatial resolution of HST/WFPC2, typical globular clusters (Harris 1996) begin to be resolved for less 

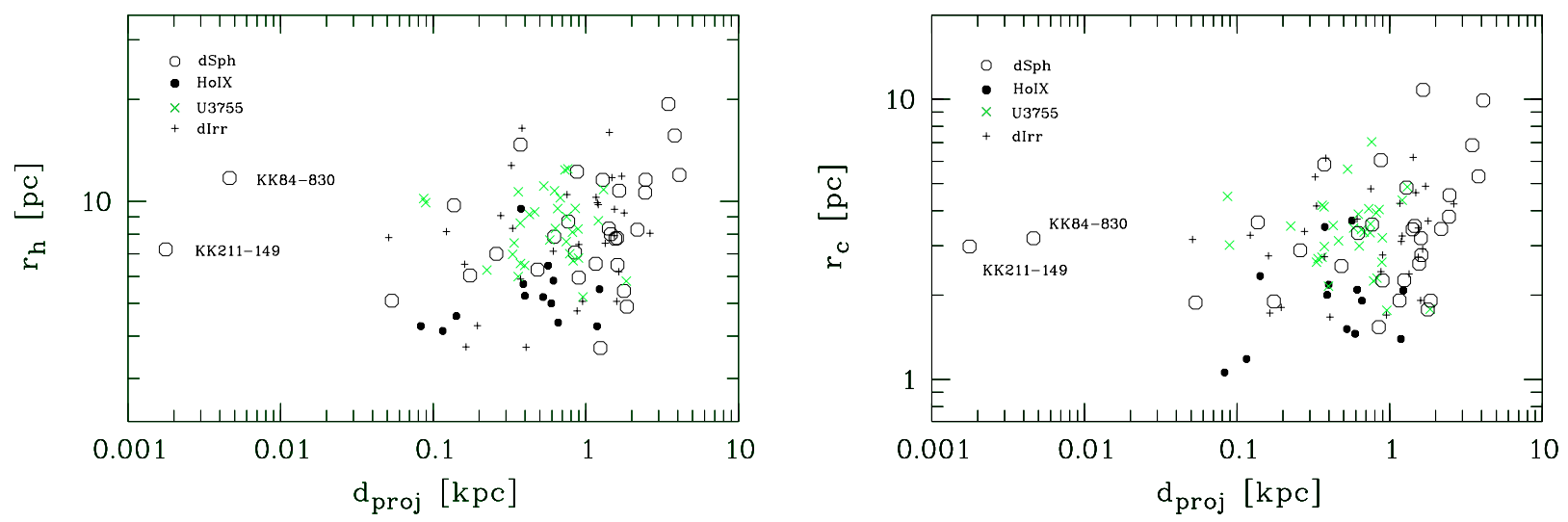

Fig. 8. Left panel: half-light radius of our sample GCCs versus their projected galactocentric distance. Right panel: core radius of GCCs versus their projected galactocentric distances. The data for the two richest GCC systems, the dIrr galaxies UGC 3755 and Holmberg IX, are marked by different symbols.

distant galaxies than $D \sim 10 \mathrm{Mpc}$. Using our King-profile approximation routine we measure structural parameters for all our sample GCCs. Figure 8 shows half-light radii, $r_{\mathrm{h}}$, and core radii, $r_{\mathrm{c}}$ of GCCs as a function of their projected galactocentric distance. Both panels show a trend of increasing half-light and core radius as a function of increasing galactocentric distance. These correlations are, however, driven by the outermost GCCs. Spectroscopy is necessary to measure their radial velocities and test whether these objects are genuine globular clusters or resolved background galaxies. If we consider GCCs with projected distances less than $\sim 1 \mathrm{kpc}$ to avoid potential contamination by background sources (see Fig. 7b), we find only tentative evidence for a $r_{\mathrm{c}}-d_{\text {proj }}$ correlation. With the same radial constraint we find no correlation between half-light radius and galactocentric distance.

Such correlations exist for half-light radii and core radii of Galactic and Large Magellanic Cloud (LMC) globular clusters (van den Bergh 2000; de Grijs et al. 2002; van den Bergh $\&$ Mackey 2004). We find that at a given galactocentric distance our sample GCCs have on average a factor of $\sim 5$ larger half-light radii than LMC globular clusters. This might be due to the higher mass of LMC, which has a stronger tidal field in which destruction processes are enhanced compared to our sample dIrr galaxies. The current dataset reveals no difference between the average structural parameter distribution of GCCs in dIrr and dSph galaxies.

We detect two GCCs in dSph galaxies at small galactocentric radii with structural parameters significantly larger than what one would expect from the extrapolation of the remaining sample (KK84-830 and KK211-149). These clusters might be fainter analogues of nuclear star clusters found in dwarf elliptical galaxies (Durrell et al. 1996), which spiraled into the cores of their host galaxies through the process of dynamical friction and orbital decay (Lotz et al. 2001). These two GCCs are among the brightest objects in our sample $\left(M_{V}=-9.7\right.$ and $-7.8 \mathrm{mag}$, see Table 2 ), but they are significantly larger and fainter than nuclear star clusters in late-type spiral galaxies, which have typical sizes between $r_{\mathrm{h}} \approx 2$ and $10 \mathrm{pc}$ and magnitudes between $M_{V} \approx-10$ and -13 mag (Böker et al. 2004). These two nuclear GCCs are also much smaller than the cores of ultra-compact dwarf galaxies, which were recently discovered in the Fornax galaxy cluster (Hilker et al. 1999; Drinkwater et al. 2003).

We also consider structural parameters of the newly discovered population of faint and extended star clusters in lenticular galaxies, termed "faint fuzzies" (Brodie \& Larsen 2002), which have typical half-light radii $r_{\mathrm{h}}>7 \mathrm{pc}$ and magnitudes fainter than $M_{V}=-7.5$ (see Fig. 9). In fact we find that roughly half of our sample is consistent with their magnitudes and structural parameters. The colors of these "faint fuzzy" clusters are around $(V-I)_{0} \approx 1.3$. We find several faint and extended GCCs in our sample with very similar colors. However, the majority of our sample are bluer and have colors typical of the red subpopulation with a mean at $(V-I)_{0} \approx 1.0 \mathrm{mag}$. We note that the most extended globular clusters in the Local Group spirals and the Magellanic Clouds resemble these faint fuzzies as well.

Figure 9 shows the distribution of our GCCs in the halflight radius versus luminosity plane. A large fraction of GCCs exhibit luminosities and half-light radii consistent with "faint fuzzy" star clusters. The majority of GCCs, however, fall below the relation found by van den Bergh \& Mackey (2004), where almost all of the Galactic globular clusters reside. Moreover, the figure shows that some GCCs, primarily those in dSph galaxies, show luminosities and structural parameters similar to those found for NGC 2419 and $\omega$ Cen, two atypically extended Galactic halo globular clusters (van den Bergh \& Mackey 2004).

\subsection{Any compact star clusters in Holmberg IX?}

We find that GCCs in the dIrr galaxy Holmberg IX are on average more compact than those situated in $\mathrm{dSph}$ and other dIrr galaxies (see Fig. 8). Almost all Holmberg IX GCCs are blue with a mean $(V-I)_{0}=0.45 \pm 0.16$ and are likely to be young $(t \lesssim 1 \mathrm{Gyr}$ ). The youth of these clusters could be the reason for their compactness, as cluster destruction processes had not have enough time to significantly alter their sizes and/or the initial conditions during cluster formation in Holmberg IX were different from those in other galaxies, for instance due to significantly higher ambient pressure. 


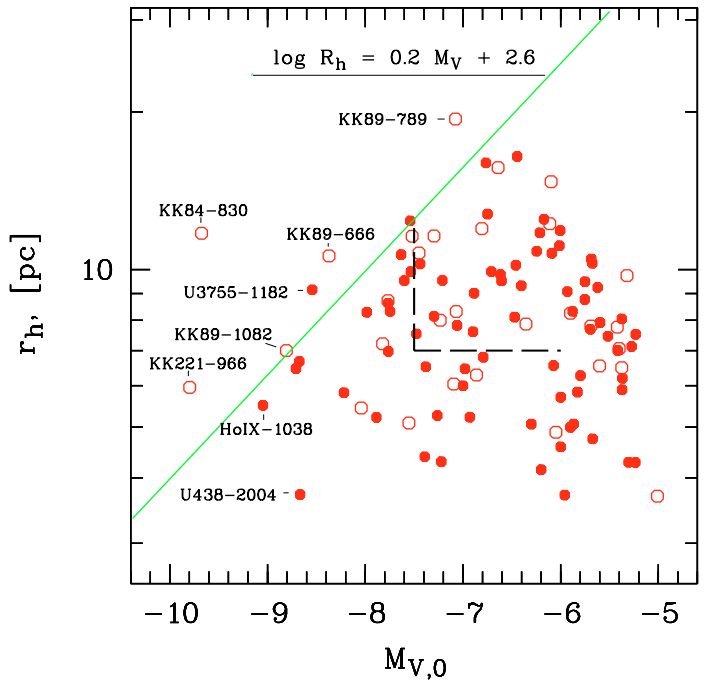

Fig. 9. Half-light radii of GCCs in dSph (open circles) and dIrr galaxies (solid dots) versus their luminosities, $M_{V, 0}$. The figure shows that almost all GCCs lie below the relation for Galactic globular clusters (solid line, see van den Bergh \& Mackey 2004), except some GCC in dSphs and one GCC in UGC3755. The most interesting cases of bright and compact GCCs in dIrrs and bright and diffuse GCCs in $\mathrm{dSphs}$ are labeled. Roughly half of GCCs is consistent with luminosities and strucrural parameters of the "faint fuzzies" of Brodie \& Larsen (2002), whose location is indicated by two dashed lines $\left(M_{V}>-7.5\right.$ and $\left.r_{\mathrm{h}}>7 \mathrm{pc}\right)$.

In a study of structural parameters of LMC globular clusters, de Grijs et al. (2002) found that older clusters exhibit a much greater spread in core radii than do their younger counterparts. If the increased spread is due to advanced dynamical evolution, one would expect a smaller spread in sizes of old globular clusters in less massive galaxies. We will test this hypothesis once accurate spectroscopic ages and chemical compositions become available.

\section{Discussion}

\subsection{Extended globular clusters}

There is increasing evidence in the current literature that lowmass galaxies host a significant population of faint and extended globular clusters. M 33 is the most prominent example (Chandar et al. 2001, 2004). Different physical mechanisms tend to destroy clusters with time: evaporation, disk shocking, tidal shock heating, dynamical friction, etc. (e.g. Gnedin \& Ostriker 1997; Surdin \& Arkhipova 1998; Fall \& Zhang 2001). The efficiency of tidal disruption increases significantly with the presence of a bulge component (Gnedin \& Ostriker 1997). M 33, as well as the dwarf galaxies in our sample, have no significant bulge components and faint and extended globular clusters are able to survive significantly longer than in the Milky Way or M 31. Consequently, there are no indications of an excess population of faint and extended globular clusters in the Milky Way or the inner halo of M 31 (Harris 2001; Barmby et al. 2001; van den Bergh \& Mackey 2004), as the efficiency of cluster disruption drops steeply with galactocentric distance.
If accretion of globular clusters plays an important role in the assembly of outer globular cluster systems in massive galaxies, one should expect similarities in magnitudes and structural parameters between clusters in our sample dwarf galaxies and the outskirts of the Local Group spirals. Indeed, most of our GCCs have similar structural parameters and luminosities to the most extended Galactic and M 31 globular clusters, which are located at large galactocentric distances. This hints at the accretion of such extended clusters from satellite dwarf galaxies (see also Forbes et al. 2004). However, most of the outer halo Galactic globular clusters are metalpoor $([\mathrm{Z} / \mathrm{H}] \lesssim-1)$ and old $(t \gtrsim 10 \mathrm{Gyr})$. If the accretion scenario for the outer-halo Galactic globular clusters is correct, then they must have formed early, perhaps in Searle-Zinn-type proto-galactic clumps (see Searle \& Zinn 1978).

It was recently found that the chemical composition of LMC globular clusters is not entirely reflected in the globular cluster systems of Local Group spirals (e.g. Puzia et al. 2005). Hence, the scenario of the assembly of their outer-halo globular cluster systems by accretion of LMC-type globular clusters seems less likely. A detailed spectroscopic investigation of globular clusters in dwarf galaxies will certainly help to constrain the picture of globular cluster accretion from satellite dwarf galaxies, and will also provide insight into the assembly history of globular cluster systems in giant elliptical galaxies.

\subsection{Star clusters in dwarf galaxies}

Our sample consists of the lowest-mass nearby dwarf galaxies and is representative and homogeneous enough to study the presence of GCCs as a function of general galaxy properties, such as morphological type. The faintest galaxies in our sample are $\mathrm{dSphs}$, with mean surface brightnesses as faint as $\mu_{\mathrm{B}} \approx 24.4$ mag/arcsec ${ }^{2}$ (see Table 2). Roughly half of our dIrr galaxies are in the same magnitude range. The detection rate of GCCs in these faint dIrr is $\sim 2$ times lower than in dSphs (and the other higher surface brightness dIrrs). Only 5 out of 19 faint dIrr galaxies contain GCCs, whereas 10 of $18 \mathrm{dSphs}$ harbor GCCs at similar host galaxy luminosities. It is not known what causes this discrepancy. We speculate that dSph galaxies turned their gas reservoir earlier and more efficiently into stars than did dIrr galaxies of similar luminosity. This was perhaps due to a higher virial density (from stars, gas and dark matter) and higher ambient pressure in the early environments of dSph galaxies. Another reason might be the different stellar $M / L$ ratio in dSph and dIrr.

It should be mentioned that our sample dSphs reveal an outstanding feature: in contrast to dIrr galaxies, $\sim 60 \%$ of dSphs with GCCs have cluster candidates located near their galaxy center. These GCCs are bright and compact $\left(-7<M_{V, 0}<-10\right.$, $\left.r_{\mathrm{c}} \approx 2-3 \mathrm{pc}\right)$, similar to those found at the center of nucleated Virgo dEs (Lotz et al. 2004).

It was shown in Sect. 4.3 that the surface density profiles of globular cluster systems in $\mathrm{dSph}$ galaxies are steeper than those in dIrrs. In general, we find that GCC systems of dSph galaxies are more spatially concentrated than in dIrr galaxies. The red sub-population of GCCs in dIrrs shows the "flattest" 
profile. Blue GCCs in dIrr galaxies, on the other hand, tend to reside in central regions of their host galaxies, but they are still less concentrated than GCCs in dSphs. This difference implies that globular cluster formation and/or evolution histories in both galaxy types were spatially not alike. Globular clusters presumably form where gas is undergoing agitated star formation. With respect to star formation histories, both $\mathrm{dSphs}$ and dIrrs appear to be inhomogeneous classes of galaxies (e.g. Mateo 1998). The difference in GCC surface density profiles might reflect a bias of powerful star formation events towards the center of dSph and/or more efficient orbital decay of star clusters in these galaxies (e.g. Lotz et al. 2001).

Both the dIrr and dSph galaxies share a sub-population of red globular clusters with very similar mean $(V-I)_{0}$ colors. In addition to this GCC population, dIrr galaxies host a very blue population of clusters, which are likely to be younger. The bimodality of the GCC color distribution in dIrr galaxies implies two different episodes and/or mechanisms of cluster formation. Similar GCC colors to those in dSphs are found for GCCs in $\mathrm{dE}$ galaxies (see Sect. 4.1). There is, however, a very puzzling offset of $\Delta(V-I)_{0} \approx 0.1$ between GCCs in $\mathrm{dE}$ and dSph galaxies, where GCCs in $\mathrm{dE}$ are bluer. This can be interpreted as the result of lower metallicities and/or younger ages.

The question about this age and/or metallicity difference is difficult to answer. However, one can consider the globular cluster system of the Fornax dSph galaxy in the Local group as a first reference to learn more about ages and metallicities of field and cluster stellar populations. Red giant branches of all globular clusters in Fornax dSph show steeper slopes than the mean RGB slope of the field stellar population (e.g. Buonanno et al. 1998, 1999). Using RGBs of Galactic globular clusters as a reference, all globular clusters in Fornax dSph were found to have low metallicities. This was confirmed by a spectroscopic study of their integrated-light (Strader et al. 2003), which found a mean metallicity of $[\mathrm{Fe} / \mathrm{H}] \approx-1.8$ and old ages. First attempts to obtain spectroscopic age and metallicity estimates for globular clusters in dEs are on the way (Miller et al. 2004).

From a study of surface brightness fluctuations, Jerjen et al. (2004) showed that Virgo dE galaxies follow a galaxy metallicity-luminosity relation. Virgo dEs should, therefore, be expected to have on average $\sim 0.7$ dex higher metallicities compared to the 100 times fainter LG dSph galaxies. Assuming old ages for their GCCs in dEs, Lotz et al. (2004) find a correlation between the mean globular cluster color and host galaxy luminosity, which implies a globular cluster metallicity-galaxy luminosity relation of the form $\left\langle Z_{\mathrm{GCC}}\right\rangle \propto L_{B}^{0.22 \pm 0.05}$. This relation implies bluer globular clusters colors in fainter galaxies. Globular clusters have generally bluer colors than their host galaxies (Ashman \& Zepf 1998), and the two relations suggest that this offset is present at all galaxy luminosities. According to those relations, globular clusters in dEs are expected to have higher metallicities than in $\mathrm{dSph}$ galaxies. Therefore, younger ages might be responsible for the bluer colors of globular clusters in $\mathrm{dE}$ galaxies.

It is important to continue the study of globular cluster systems in nearby low-mass galaxies and to compare their properties as a function of different host galaxy properties, e.g. morphological type and environmental density.
Acknowledgements. M.E.S. and D.I.M. are partially supported by an RFBR 04-02-16115 grant. T.H.P. is supported by an ESA Research Fellowship, which is gratefully acknowledged. D.I.M. thanks the Russian Science Support Foundation. M.E.S kindly thanks I. D. Karachentsev for initiating the beginning of a work on searches of globular clusters in dSph galaxies and Vladimir Surdin for useful discussions. We thank Jennifer Lotz for providing data of globular cluster candidates in dwarf elliptical galaxies in electronic form. T.H.P. thanks Rupali Chandar, Nicole Homeier, and Jennifer Lotz for useful discussions. We thank the anonymous referee for helpful comments.

\section{References}

Ashman, K. A., Bird, C. M., \& Zepf, S. E. 1994, AJ, 108, 2348

Ashman, K. M., \& Zepf, S. E. 1998, Globular cluster systems (Cambridge, UK, New York: Cambridge University Press)

Ashman, K. M., \& Zepf, S. E. 2001, AJ, 122, 1888

Barmby, P., Huchra, J. P., \& Brodie, J. P. 2001, AJ, 121, 1482

Billett, O. H., Hunter, D. A., \& Elmegreen, B. G. 2002, AJ, 123, 1454

Böker, T., Sarzi, M., McLaughlin, D. E., et al. 2004, AJ, 127, 105

Bothun, G., Impey, C., \& McGaugh, S. 1997, PASP, 109, 745

Boyce, P. J., Minchin, R. F., Kilborn, V. A., et al. 2001, ApJ, 560, L127

Brodie, J. P., \& Larsen, S. S. 2002, AJ, 124, 1410

Bruzual, G., \& Charlot, S. 2003, MNRAS, 344, 1000

Buonanno, R., Corsi, C., Zinn, R., et al. 1998, ApJ, 501, L33

Buonanno, R., Corsi, C., Fusi Pecci, F., \& Zinn, R. 1999, AJ, 118, 1671

Chandar, R., Bianchi, L., \& Ford, H. C. 2001, A\&A, 366, 498

Chandar, R., Whitmore, B., \& Lee, M. G. 2004, ApJ, 611, 220

de Grijs R., Gilmore, G. F., Mackey, A. D., et al. 2002, MNRAS, 337, 597

Davies, J. I., \& Phillipps, S. 1988, MNRAS, 233, 553

Drinkwater, M. J., Gregg, M. D., Hilker, M., et al. 2003, Nature, 423, 519

Durrell, P. R., Harris, W. E., Geisler, D., \& Pudritz, R. E. 1996, AJ, 112,972

Elmegreen, B. G. 2002, ApJ, 577, 206

Elmegreen, B. G., \& Efremov, Y. N. 1997, ApJ, 480, 235

Fall, S. M., \& Zhang, Q. 2001, ApJ, 561, 751

Ferguson, H. C., \& Binggeli, B. 1994, A\&AR, 6, 67

Forbes, D. A., Strader, J., \& Brodie, J. P. 2004, AJ, 127, 3394

Gnedin, O. Y., \& Ostriker, J. P. 1997, ApJ, 474, 223

Goudfrooij, P., Gilmore, D., Whitmore, B. C., \& Schweizer, F. 2004, ApJ, 613, L121

Heidt, J., Appenzeller, I., Gabasch, A., et al. 2003, A\&A, 398, 49

Harris, W. E. 1986, AJ, 91, 822

Harris, W.E. 1996, AJ, 112, 1487, for the 2003 update see

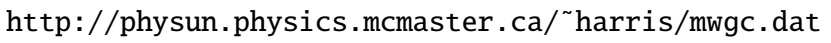

Harris, W. E. 2001, Saas-Fee Advanced Course 28: Star Clusters, 223

Hilker, M., Infante, L., Vieira, G., Kissler-Patig, M., \& Richtler, T. 1999, A\&AS, 134, 75

Hodge, P. W., Skelton, B. P., \& Ashizawa, J. 2002, An Atlas of Local Group Galaxies, ed. P. W. Hodge, B. P. Skelton, \& J. Ashizawa, University of Washington, Astronomy Department, Seattle, USA, Astrophysics and Space Science Library, 221 (Dordrecht: Kluwer Academic Publishers)

Holtzman, J. A., Burrows, C. J., Casertano, S., et al. 1995, PASP, 107, 1065

Hunter, D. A., \& Elmegreen, B. G. 2004, AJ, 128, 2170

Impey, C., \& Bothun, G. 1997, ARA\&A, 35, 267

James, P. A., Shane, N. S., Knapen, J. H., Etherton, J., \& Percival, S. M. 2005, A\&A, 429, 851

Jerjen, H., Binggeli, B., \& Barazza, F. D. 2004, AJ, 127, 771 
Jordán, A., Côté, P., West, M. J., \& Marzke, R. O. 2002, ApJ, 576, L113

Karachentsev, I. D., Karachentseva, V. E., Dolphin, A. E., et al. 2000a, A\&A, 363, 117

Karachentsev, I. D., Sharina, M. E., Grebel, E. K., et al. 2000b, ApJ, 542,128

Karachentsev, I. D., Sharina, M. E., Dolphin, A. E., et al. 2001a, A\&A, 379, 407

Karachentsev, I. D., Sharina, M. E., Dolphin, A. E., et al. 2001b, A\&A, 375, 359

Karachentsev, I. D., Sharina, M. E., Dolphin, A. E., et al. 2003, A\&A, 398, 467

Karachentsev, I. D., Karachentseva, V. E., Huchtmeier, W. K., \& Makarov, D. I. 2004, AJ, 127, 2031

King, I. 1962, AJ, 67, 471

Kissler-Patig, M. 2000, Rev. Mod. Astron., 13, 13

Klypin, A., Kravtsov, A. V., Valenzuela, O., \& Prada, F. 1999, ApJ, 522, 82

Kravtsov, A. V., Gnedin, O. Y., \& Klypin, A. A. 2004, ApJ, 609, 482

Kundu, A., \& Whitmore, B. C. 2001a, AJ, 121, 2950

Kundu, A., \& Whitmore, B. C. 2001b, AJ, 122, 1251

Lada, C. J., \& Lada, E. A. 2003, ARA\&A, 41, 57

Larsen, S. S., \& Richtler, T. 2000, A\&A, 354, 836

Larsen, S. S., Brodie, J. P., Huchra, J. P., Forbes, D. A., \& Grillmair, C. J. 2001, AJ, 121, 2974

Lotz, J. M., Telford, R., Ferguson, H. C., et al. 2001, ApJ, 552, 572

Lotz, J. M., Miller, B. W., \& Ferguson, H. C. 2004, ApJ, 613, 262

Makarova, L. N. 1999, A\&A, 139, 491

Makarova, L. N., Karachentsev, I., Takalo, L. O., Heinaemaeki, P., \& Valtonen, M. 1998, A\&AS, 128, 459
Marlowe, A. T., Meurer, G. R., \& Heckman, T. M. 1999, ApJ, 522, 183

Mateo, M. 1998, ARA\&A, 36, 435

Miller, B., Lotz, J., Hilker, M., et al. 2004, A\&AS Meet. Abstracts, 204

Minniti, D., Meylan, G., \& Kissler-Patig, M. 1996, A\&A, 312, 49

Pelupessy, F. I., van der Werf, P. P., \& Icke, V. 2004, A\&A, 422, 55

Puzia, T. H., Kissler-Patig, M., Brodie, J. P., \& Huchra, J. P. 1999, AJ, 118,2734

Puzia, T. H., Kissler-Patig, M., Thomas, D., et al. 2004, A\&A, 415, 123

Puzia, T. H., Perrett, K. M., \& Bridges, T. J. 2005, A\&A, 434, 909

Searle, L., \& Zinn, R. 1978, ApJ, 225, 357

Secker, J. 1992, AJ, 104, 1472

Schlegel, D. J., Finkbeiner, D. P., \& Davis, M. 1998, ApJ, 500, 525

Silverman, B. W. 1986, Density Estimation for Statistics and Data Analysis, Chap and Hall/CRC Press, Inc.

Stetson, P. B. 1987, PASP, 99, 191

Strader, J., Brodie, J. P., Forbes, D. A., Beasley, M. A., \& Huchra, J. P. 2003, AJ, 125, 1291

Surdin, V. G., \& Arkhipova, N. A., 1998, Russian Astronomy Letters, 24, 343

van den Bergh, S. 2000, ApJ, 530, 777

van den Bergh, S., \& Mackey, A. D. 2004, MNRAS, 354, 713

de Vaucouleurs, G., de Vaucouleurs, A., Corwin, H. G. Jr., et al. 1991, Third Reference Catalogue of bright galaxies (Berlin: Springer) Weilbacher, P. M. 2002, Ph.D. Thesis

Whitmore, B. C., Zhang, Q., Leitherer, C., et al. 1999, AJ, 118, 1551

Yun, M. S., Ho, P. T. P., \& Lo, K. Y. 1994, Nature, 372, 530 
M. E. Sharina et al.: HST imaging of globular cluster candidates in nearby LSB dwarf galaxies, Online Material $p 1$

\section{Online Material}


M. E. Sharina et al.: HST imaging of globular cluster candidates in nearby LSB dwarf galaxies, Online Material p 2

Table 1. Low surface brightness galaxies in nearby groups and in the field, which were searched for globular cluster candidates. Columns of the table contain the following data: (1) Galaxy name, (2) equatorial coordinates (J2000), (3) morphological type according to RC3 (de Vaucouleurs et al. 1991), (4) distance in Mpc, (5) integrated absolute $V$ magnitude (indices refer to 0: this work; K0: Karachentsev et al. 2000b; K1a: Karachentsev et al. 2000a; K1b: Karachentsev et al. 2001a; K1c: Karachentsev et al. 2001b; RC3: de Vaucouleurs et al. 1991; M98: Makarova et al. 1998; M99: Makarova 1999), (6) logarithmic surface gas density of neutral hydrogen in $M_{\odot} / \mathrm{kpc}^{2}$, (7) semi-major axis diameter in kpc, (8) mean surface brightness in $B$-band, (9) number of globular cluster candidates, including (10) number of GCCs located outside the isophote of constant surface brightness $\mu_{B}=26.5 \mathrm{mag} / \mathrm{arcsec}^{2}$.

\begin{tabular}{|c|c|c|c|c|c|c|c|c|c|c|}
\hline Name & RA (2000) & $\operatorname{Dec}(2000)$ & $T$ & Dist & $M_{V}$ & $\log \Sigma_{H I}$ & $R_{\mathrm{kpc}}$ & $S B_{\text {mean }}$ & $N_{\mathrm{GCC}}$ & $N_{\text {out }}$ \\
\hline \multicolumn{11}{|l|}{ M 81 group } \\
\hline KDG52 & 082356 & +710146 & 10 & 3.55 & $-11.71_{0}$ & 7.0 & 1.3 & 25.5 & 0 & 0 \\
\hline DDO53 & 083407 & +661045 & 10 & 3.56 & $-13.74_{R C 3}$ & 7.3 & 1.7 & 24.1 & 1 & 0 \\
\hline A $0952+69$ & 095729 & +691620 & 10 & 3.87 & $-11.84_{0}$ & $\ldots$ & 2.0 & 26.5 & 0 & 0 \\
\hline $\mathrm{BK} 3 \mathrm{~N}$ & 095349 & +685809 & 10 & 4.02 & $-9.59_{0}$ & $\cdots$ & 0.5 & 25.6 & 1 & 1 \\
\hline KDG73 & 105255 & +693245 & 10 & 3.70 & $-11.31_{0}$ & 7.0 & 0.6 & 24.5 & 1 & 1 \\
\hline FM1 & 094510 & +684554 & -3 & 3.42 & $-11.04_{K 1 c}$ & $\ldots$ & 0.9 & 25.7 & 0 & 0 \\
\hline KK77 & 095010 & +673024 & -3 & 3.48 & $-12.21_{K 1 a}$ & $\ldots$ & 2.4 & 26.4 & 3 & 0 \\
\hline KDG61, KK81 & 095703 & +683530 & -1 & 3.60 & $-13.58_{K 1 a}$ & $\ldots$ & 2.5 & 25.4 & 1 & 0 \\
\hline KKH57, HS108 & 100016 & +631106 & -3 & 3.93 & $-10.90_{K 1 c}$ & $\ldots$ & 0.7 & 25.3 & 0 & 0 \\
\hline KDG63, KK83 & 100507 & +663318 & -3 & 3.50 & $-12.82_{K 1 a}$ & $\ldots$ & 1.7 & 25.5 & 1 & 0 \\
\hline KDG64, KK85 & 100702 & +674939 & -3 & 3.70 & $-13.24_{K 1 a}$ & $\ldots$ & 2.0 & 25.1 & 0 & 0 \\
\hline DDO78, KK89 & 102628 & +673924 & -3 & 3.72 & $-12.75_{K 1 a}$ & $\ldots$ & 2.1 & 25.8 & 2 & 0 \\
\hline BK6N, KK91 & 103432 & +660042 & -3 & 3.85 & $-11.93_{K 1 a}$ & $\ldots$ & 1.2 & 25.5 & 2 & 0 \\
\hline Garland & 100342 & +684136 & 10 & 3.7 & $\ldots$ & 7.3 & 4.3 & & 1 & $\ldots$ \\
\hline Holmberg IX & 095732 & +690235 & 10 & 3.7 & $-13.8_{0}$ & 7.9 & 2.5 & 24.8 & 14 & 0 \\
\hline \multicolumn{11}{|l|}{ Sculptor group } \\
\hline E410-005, KK3 & 001531 & -321048 & -1 & 1.92 & $-12.11_{K 0}$ & $\ldots$ & 0.7 & 24.0 & 0 & 0 \\
\hline KDG2, E540-030 & 004921 & -180428 & -1 & 3.40 & $-12.00_{0}$ & $\ldots$ & 1.2 & 25.3 & 1 & 1 \\
\hline E294-010, PGC1641 & 002633 & -415120 & -3 & 1.92 & $-11.40_{0}$ & $\ldots$ & 0.6 & 24.2 & 1 & 0 \\
\hline E540-032, FG24 & 005025 & -195425 & -3 & 3.42 & $-11.84_{0}$ & $\ldots$ & 1.3 & 25.6 & 0 & 0 \\
\hline KK27 & 032106 & -661922 & -3 & 3.98 & $-12.32_{0}$ & $\ldots$ & 1.4 & 24.9 & 1 & 0 \\
\hline $\mathrm{Sc} 22$ & 002352 & -244218 & -3 & 4.21 & $-11.10_{0}$ & $\ldots$ & 1.1 & 26.0 & 3 & 2 \\
\hline DDO6 & 004949 & -210058 & 10 & 3.34 & $-12.92_{R C 3}$ & 6.8 & 1.7 & 24.5 & 0 & 0 \\
\hline \multicolumn{11}{|l|}{ CVn I cloud } \\
\hline KK166 & 124913 & +353645 & -3 & 4.74 & $-11.29_{0}$ & $\ldots$ & 1.0 & 25.2 & 0 & 0 \\
\hline DDO113, KDG90 & 121458 & +361308 & 10 & 2.86 & $-12.67_{0}$ & & 1.2 & 25.1 & 2 & 1 \\
\hline U7605 & 122839 & +354305 & 10 & 4.43 & $-13.88_{M 98}$ & 7.3 & 1.4 & 23.4 & 1 & 0 \\
\hline KK109 & 114711 & +434019 & 10 & 4.51 & $-10.19_{0}$ & 6.9 & 0.8 & 25.9 & 1 & 1 \\
\hline U7298 & 121629 & +521338 & 10 & 4.21 & $-12.54_{M 99}$ & 7.2 & 1.3 & 24.5 & 1 & 0 \\
\hline U8308, DDO167 & 131322 & +461918 & 10 & 4.19 & $-12.48_{M 99}$ & 7.2 & 1.3 & 24.0 & 4 & 2 \\
\hline U8833 & 135449 & +355015 & 10 & 3.19 & $-12.73_{M 98}$ & 7.4 & 0.8 & 23.5 & 0 & 0 \\
\hline \multicolumn{11}{|l|}{ Cent A group } \\
\hline KK211 & 134206 & -451218 & -5 & 3.58 & $-12.58_{0}$ & $\ldots$ & 0.8 & 24.1 & 2 & 0 \\
\hline KK213 & 134336 & -434609 & -3 & 3.63 & $-11.12_{0}$ & $\ldots$ & 0.6 & 25.5 & 0 & 0 \\
\hline KK217 & 134617 & -454105 & -3 & 3.84 & $-12.18_{0}$ & $\cdots$ & 0.7 & 24.7 & 0 & 0 \\
\hline KK221 & 134846 & -465949 & -3 & 3.98 & $-11.96_{0}$ & $\ldots$ & 1.7 & 27.0 & 5 & 0 \\
\hline E269-37, KK179 & 130334 & -463503 & -3 & 3.48 & $-12.57_{0}$ & $\ldots$ & 0.8 & 24.0 & 0 & 0 \\
\hline KK200 & 132436 & -305820 & 9 & 4.63 & $-12.74_{0}$ & 6.6 & 1.8 & 25.5 & 1 & 0 \\
\hline E444-84 & 133720 & -280246 & 10 & 4.61 & $-13.63_{0}$ & 7.6 & 1.7 & 24.0 & 0 & 0 \\
\hline \multicolumn{11}{|l|}{ N3115 group } \\
\hline KK84 & 100534 & -074457 & -3 & 9.7 & $-14.40_{0}$ & $\ldots$ & 4.1 & 25.4 & 8 & 2 \\
\hline \multicolumn{11}{|l|}{ Field } \\
\hline KKR25 & 161348 & +542216 & 10 & 1.86 & $-10.45_{K 1 b}$ & 6.7 & 0.6 & 25.0 & 0 & 0 \\
\hline U8508 & 133044 & +545436 & 10 & 2.56 & $-13.42_{M 99}$ & 7.3 & 1.3 & 23.6 & 0 & 0 \\
\hline DDO190, U9240 & 142444 & +443133 & 10 & 2.79 & $-14.37_{R C 3}$ & 7.4 & 1.5 & 22.9 & 1 & 0 \\
\hline E379-07, KK112 & 115443 & -333329 & 10 & 5.22 & $-12.28_{0}$ & 6.9 & 1.7 & 25.1 & 3 & 0 \\
\hline E321-014 & 121350 & -381353 & 10 & 3.19 & $-13.18_{0}$ & 6.8 & 1.3 & 24.0 & 0 & 0 \\
\hline KKH5 & 010733 & +512625 & 10 & 4.26 & $-12.64_{0}$ & 7.1 & 0.7 & 23.7 & 0 & 0 \\
\hline KKH34, Mai13 & 055941 & +732539 & 10 & 4.61 & $-12.70_{0}$ & 6.9 & 1.2 & 24.7 & 0 & 0 \\
\hline KКH98 & 234534 & +384304 & 10 & 2.45 & $-11.28_{0}$ & 7.1 & 0.7 & 25.0 & 0 & 0 \\
\hline KK16 & 015521 & +275715 & 10 & 4.74 & $-12.81_{0}$ & 6.7 & 1.1 & 23.9 & 0 & 0 \\
\hline KK17 & 020010 & +284957 & 10 & 4.72 & $-11.95_{0}$ & 6.7 & 0.8 & 24.3 & 0 & 0 \\
\hline KKH18 & 030306 & +334140 & 10 & 4.43 & $-12.84_{0}$ & 7.3 & 0.9 & 23.8 & 0 & 0 \\
\hline E489-56, KK54 & 062617 & -261556 & 10 & 4.99 & $-13.51_{0}$ & 7.4 & 0.9 & 22.8 & 0 & 0 \\
\hline E490-17, PGC19337 & 063757 & -255959 & 10 & 4.23 & $-14.91_{0}$ & 6.9 & 2.1 & 23.5 & 5 & 0 \\
\hline U3755 & 071352 & +103119 & 10 & 5.22 & $-15.36_{M 99}$ & 6.9 & 2.6 & 23.4 & 32 & 0 \\
\hline KK65 & 074231 & +163340 & 10 & 4.51 & $-13.32_{0}$ & 7.1 & 1.2 & 23.3 & 1 & 0 \\
\hline U4115 & 075702 & +142327 & 10 & 5.49 & $-14.12_{0}$ & 7.4 & 2.9 & 24.8 & 3 & 0 \\
\hline KKH86 & 135434 & +041435 & 10 & 2.61 & $-11.19_{0}$ & 6.6 & 0.5 & 24.5 & 0 & 0 \\
\hline UA438, E470-18 & 232628 & -322326 & 10 & 2.23 & $-11.94_{0}$ & 7.4 & 1.0 & 23.2 & 2 & 0 \\
\hline KKH98 & 234534 & +384304 & 10 & 2.45 & $-11.28_{0}$ & 7.1 & 0.8 & 25.0 & 0 & 0 \\
\hline E321-014 & 121350 & -381353 & 10 & 3.19 & $-13.18_{0}$ & 6.8 & 1.3 & 24.0 & 0 & 0 \\
\hline
\end{tabular}


M. E. Sharina et al.: HST imaging of globular cluster candidates in nearby LSB dwarf galaxies, Online Material p 3

Table 2. List of globular cluster candidates (GCCs) in nearby LSB dwarf galaxies. The columns contain the following data: Identifier of each cluster, composed of the (name of its host galaxy)-(WFPC2 chip)-(cluster numbering), $X, Y$ coordinates derived from the WFPC2 frames, equatorial coordinates (J2000.0), half-light radius $r_{\mathrm{h}}$ in parsecs, apparent axial ratio $e=1-b / a$, integrated absolute $V$ magnitude (corrected for Galactic extinction using Schlegel et al. 1998, maps) and corresponding error, integrated absolute $V-I$ color (corrected for Galactic extinction) and corresponding error, and the projected separation from the center of its host galaxy $d_{\text {proj }}$ in kiloparsecs. The numbers of GCCs located outside the isophote of constant surface brightness $\mu_{B} \sim 26.5 \mathrm{mag} / \operatorname{arcsec}^{2}$ of the host galaxy are marked by an asterisk symbol ( ${ }^{*}$ ).

\begin{tabular}{|c|c|c|c|c|c|c|c|}
\hline GCC & $X, Y$ & RA (J2000) Dec & $r_{\mathrm{h}}$ & $e$ & $M_{V, 0}$ & $(V-I)_{0}$ & $d_{\text {proj }}$ \\
\hline DDO53-3-1120 & $291.022,208.595$ & $083404.1+661023$ & 6.7 & 0.2 & $-5.88 \pm 0.07$ & $1.39 \pm 0.10$ & 0.33 \\
\hline BK3N-2-863* & $546.324,579.284$ & $095400.1+685854$ & 6.8 & 0.1 & $-5.23 \pm 0.08$ & $0.93 \pm 0.11$ & 1.34 \\
\hline KDG73-2-378* & $377.384,64.881$ & $105307.7+693202$ & 8.3 & 0.1 & $-5.75 \pm 0.08$ & $1.11 \pm 0.11$ & 1.54 \\
\hline KK77-4-939 & $97.291,330.684$ & $095000.3+673110$ & 3.7 & 0.3 & $-5.01 \pm 0.09$ & $0.77 \pm 0.12$ & 1.25 \\
\hline KK77-4-1162 & $138.177,568.545$ & $094956.2+673111$ & 6.5 & 0.1 & $-5.37 \pm 0.08$ & $0.82 \pm 0.11$ & 1.61 \\
\hline KK77-4-1165 & $491.903,572.199$ & $094954.8+673037$ & 7.8 & 0.0 & $-5.69 \pm 0.08$ & $1.20 \pm 0.11$ & 1.60 \\
\hline KDG61-3-1325 & $363.770,403.865$ & $095702.8+683535$ & 4.7 & 0.1 & $-7.55 \pm 0.07$ & $0.92 \pm 0.10$ & 0.05 \\
\hline KDG63-3-1168 & $347.918,329.025$ & $100507.2+663330$ & 6.0 & 0.0 & $-7.09 \pm 0.07$ & $1.07 \pm 0.10$ & 0.17 \\
\hline DDO78-1-167 & $421.025,524.751$ & $102628.3+674045$ & 7.4 & 0.1 & $-7.23 \pm 0.07$ & $0.78 \pm 0.10$ & 1.46 \\
\hline DDO78-3-1082 & $483.889,549.646$ & $102627.1+673910$ & 7.0 & 0.1 & $-8.81 \pm 0.07$ & $0.96 \pm 0.10$ & 0.26 \\
\hline BK6N-2-524 & $225.256,182.777$ & $103429.3+660129$ & 4.4 & 0.3 & $-5.40 \pm 0.08$ & $1.18 \pm 0.11$ & 0.85 \\
\hline BK6N-4-789 & $608.148,247.233$ & $103419.8+660032$ & 4.5 & 0.3 & $-5.60 \pm 0.07$ & $1.45 \pm 0.10$ & 1.16 \\
\hline Garland-1-728 & $477.284,107.876$ & $100330.8+684155$ & 3.2 & 0.1 & $-8.26 \pm 0.07$ & $0.94 \pm 0.10$ & 1.23 \\
\hline HoIX-3-866 & $384.173,122.948$ & $095737.7+690229$ & 5.2 & 0.0 & $-7.89 \pm 0.07$ & $0.54 \pm 0.10$ & 0.52 \\
\hline HoIX-3-1168 & $596.137,284.848$ & $095733.7+690214$ & 4.0 & 0.3 & $-6.00 \pm 0.08$ & $0.30 \pm 0.11$ & 0.39 \\
\hline HoIX-3-1322 & $383.979,347.673$ & $095733.7+690236$ & 4.5 & 0.3 & $-6.00 \pm 0.08$ & $0.30 \pm 0.11$ & 0.14 \\
\hline HoIX-3-1565 & $376.681,438.844$ & $095732.2+690239$ & 4.1 & 0.1 & $-5.31 \pm 0.08$ & $0.74 \pm 0.11$ & 0.08 \\
\hline HoIX-3-1664 & $378.637,471.226$ & $095731.6+690240$ & 4.1 & 0.0 & $-6.20 \pm 0.07$ & $0.37 \pm 0.10$ & 0.12 \\
\hline HoIX-3-1932 & $264.772,567.834$ & $095730.5+690254$ & 9.4 & 0.3 & $-6.61 \pm 0.07$ & $0.71 \pm 0.10$ & 0.38 \\
\hline HoIX-3-2116 & $376.956,643.274$ & $095728.5+690245$ & 5.0 & 0.0 & $-7.26 \pm 0.07$ & $0.37 \pm 0.10$ & 0.40 \\
\hline HoIX-3-2129 & $155.863,649.230$ & $095729.7+690306$ & 5.1 & 0.1 & $-5.83 \pm 0.08$ & $0.38 \pm 0.11$ & 0.61 \\
\hline HoIX-3-2158 & $200.676,656.790$ & $095729.3+690302$ & 6.2 & 0.0 & $-6.98 \pm 0.08$ & $0.30 \pm 0.11$ & 0.57 \\
\hline HoIX $-3-2373$ & $558.027,742.226$ & $095725.8+690231$ & 7.9 & 0.1 & $-6.04 \pm 0.08$ & $1.31 \pm 0.11$ & 0.61 \\
\hline HoIX-3-2376 & $322.360,744.024$ & $095727.1+690253$ & 4.8 & 0.0 & $-5.90 \pm 0.08$ & $0.30 \pm 0.11$ & 0.59 \\
\hline HoIX-3-2409 & $266.049,762.700$ & $095727.0+690259$ & 3.8 & 0.2 & $-7.39 \pm 0.07$ & $0.68 \pm 0.10$ & 0.66 \\
\hline HoIX-4-1038 & $172.803,269.307$ & $095740.0+690325$ & 5.5 & 0.1 & $-9.05 \pm 0.07$ & $0.44 \pm 0.10$ & 1.23 \\
\hline HoIX-4-1085 & $307.374,301.260$ & $095737.8+690332$ & 3.8 & 0.1 & $-5.23 \pm 0.09$ & $0.48 \pm 0.13$ & 1.18 \\
\hline E540-030-4-1183* & $150.605,590.136$ & $004920.6-180251$ & 6.2 & 0.1 & $-5.37 \pm 0.08$ & $1.24 \pm 0.11$ & 1.65 \\
\hline E294-010-3-1104 & $485.338,317.674$ & $002632.6-415110$ & 6.7 & 0.3 & $-5.32 \pm 0.07$ & $1.34 \pm 0.10$ & 0.14 \\
\hline KK027-4-721 & $435.879,190.280$ & $032110.0-661826$ & 7.5 & 0.0 & $-6.36 \pm 0.07$ & $1.15 \pm 0.10$ & 0.62 \\
\hline Sc22-2-879 & $108.743,520.881$ & $002353.3-244139$ & 12.2 & 0.2 & $-6.11 \pm 0.08$ & $1.15 \pm 0.11$ & 0.88 \\
\hline $\operatorname{Sc} 22-2-100^{*}$ & $734.953,661.836$ & $002355.5-244044$ & 8.3 & 0.0 & $-5.90 \pm 0.07$ & $0.96 \pm 0.11$ & 2.17 \\
\hline Sc22-4-106* & $598.857,570.335$ & $002344.9-244209$ & 4.9 & 0.2 & $-6.05 \pm 0.07$ & $0.96 \pm 0.11$ & 1.86 \\
\hline DDO113-2-579* & $765.664,227.122$ & $121452.8+361438$ & 7.9 & 0.2 & $-5.60 \pm 0.07$ & $1.38 \pm 0.10$ & 1.54 \\
\hline DDO113-4-690 & $368.908,76.646$ & $121454.4+361255$ & 6.5 & 0.1 & $-5.27 \pm 0.08$ & $0.99 \pm 0.11$ & 0.61 \\
\hline U7605-3-1503 & $547.917,423.740$ & $122839.9+354301$ & 12.2 & 0.3 & $-6.44 \pm 0.08$ & $1.18 \pm 0.11$ & 0.38 \\
\hline KK109-3-1200* & $538.659,754.664$ & $114708.1+434028$ & 4.4 & 0.2 & $-5.87 \pm 0.08$ & $0.92 \pm 0.11$ & 0.95 \\
\hline U7298-3-1280 & $741.363,371.228$ & $121627.3+521309$ & 4.6 & 0.1 & $-5.67 \pm 0.08$ & $0.66 \pm 0.11$ & 0.88 \\
\hline U8308-2-1198 & $145.016,739.639$ & $131327.2+461923$ & 7.5 & 0.0 & $-5.52 \pm 0.08$ & $1.08 \pm 0.12$ & 0.90 \\
\hline U8308-3-2040 & $39.610,748.843$ & $131317.0+461907$ & 9.1 & 0.1 & $-6.62 \pm 0.08$ & $1.46 \pm 0.11$ & 1.21 \\
\hline U8308-4-893* & $663.358,350.654$ & $131315.3+461932$ & 5.1 & 0.1 & $-6.30 \pm 0.07$ & $1.55 \pm 0.10$ & 1.59 \\
\hline U8308-4-971* & $699.289,438.755$ & $131314.4+461935$ & 8.3 & 0.1 & $-5.62 \pm 0.09$ & $1.20 \pm 0.12$ & 1.78 \\
\hline KK211-3-917 & $296.748,173.889$ & $134208.0-451229$ & 6.3 & 0.1 & $-6.86 \pm 0.07$ & $0.91 \pm 0.10$ & 0.48 \\
\hline KK211-3-149 & $429.726,419.984$ & $134205.6-451220$ & 6.1 & 0.2 & $-7.82 \pm 0.07$ & $0.95 \pm 0.10$ & 0 \\
\hline KK221-2-608 & $550.374,168.636$ & $134854.9-470010$ & 5.0 & 0.1 & $-8.04 \pm 0.07$ & $1.00 \pm 0.10$ & 1.78 \\
\hline KK221-2-883 & $404.756,354.161$ & $134852.8-470019$ & 8.3 & 0.1 & $-7.07 \pm 0.07$ & $1.00 \pm 0.10$ & 1.41 \\
\hline KK221-2-966 & $140.354,399.808$ & $134850.3-470010$ & 5.7 & 0.0 & $-9.80 \pm 0.07$ & $0.98 \pm 0.10$ & 090 \\
\hline KK221-2-1090 & $78.866,479.706$ & $134849.4-470014$ & 8.7 & 0.0 & $-7.77 \pm 0.07$ & $0.91 \pm 0.10$ & 0.77 \\
\hline KK221-3-1062 & $301.010,266.780$ & $134848.2-465946$ & 9.1 & 0.3 & $-6.10 \pm 0.08$ & $0.93 \pm 0.11$ & 0.37 \\
\hline KK200-3-1696 & $785.776,763.252$ & $132432.2-305811$ & 9.2 & 0.1 & $-5.68 \pm 0.09$ & $1.03 \pm 0.12$ & 1.17 \\
\hline KK84-2-785 & $173.139,447.814$ & $100535.8-074408$ & 11.6 & 0.0 & $-7.30 \pm 0.08$ & $1.00 \pm 0.11$ & 2.46 \\
\hline KK84-2-974 & $432.320,678.779$ & $100537.5-074344$ & 14.9 & 0.0 & $-6.64 \pm 0.09$ & $0.57 \pm 0.14$ & 3.82 \\
\hline KK84-3-705 & $460.100,105.800$ & $100535.7-074426$ & 9.2 & 0.1 & $-7.45 \pm 0.08$ & $1.04 \pm 0.11$ & 1.66 \\
\hline KK84-3-830 & $395.303,454.705$ & $100535.1-074460$ & 10.6 & 0.1 & $-9.68 \pm 0.07$ & $0.96 \pm 0.10$ & 0 \\
\hline KK84-3-917 & $624.322,609.065$ & $100536.5-074517$ & 10.4 & 0.1 & $-7.52 \pm 0.08$ & $1.06 \pm 0.11$ & 1.29 \\
\hline KK84-4-666 & $538.305,207.182$ & $100531.5-074504$ & 10.6 & 0.0 & $-8.37 \pm 0.07$ & $1.26 \pm 0.10$ & 2.45 \\
\hline KK84-4-789 & $290.956,379.188$ & $100530.5-074438$ & 19.4 & 0.3 & $-7.08 \pm 0.08$ & $0.88 \pm 0.12$ & 3.48 \\
\hline KK84-4-967 & $571.750,560.942$ & $100529.1-074504$ & 12.0 & 0.2 & $-6.81 \pm 0.08$ & $0.95 \pm 0.12$ & 4.10 \\
\hline U9240-3-4557 & $545.377,491.651$ & $142445.0+443136$ & 3.6 & 0.2 & $-7.22 \pm 0.07$ & $0.61 \pm 0.10$ & 0.19 \\
\hline KK112-3-976 & $447.781,261.936$ & $115443.3-333344$ & 9.1 & 0.1 & $-5.93 \pm 0.08$ & $1.25 \pm 0.11$ & 0.28 \\
\hline KK112-4-742 & $183.817,164.666$ & $115447.3-333323$ & 11.8 & 0.1 & $-6.21 \pm 0.08$ & $0.88 \pm 0.11$ & 1.48 \\
\hline KK112-4-792 & $444.689,215.677$ & $115446.6-333258$ & 15.0 & 0.1 & $-6.77 \pm 0.08$ & $1.22 \pm 0.11$ & 1.43 \\
\hline E490-017-3-1769 & $306.876,462.734$ & $063757.0-260008$ & 6.6 & 0.2 & $-7.06 \pm 0.08$ & $0.30 \pm 0.11$ & 0.05 \\
\hline E490-017-3-1861 & $246.213,480.112$ & $063757.3-260013$ & 6.4 & 0.1 & $-7.38 \pm 0.07$ & $0.87 \pm 0.10$ & 0.16 \\
\hline E490-017-3-1956 & $507.111,498.721$ & $063757.0-255948$ & 5.1 & 0.3 & $-5.37 \pm 0.09$ & $0.55 \pm 0.13$ & 0.37 \\
\hline E490-017-3-2035 & $374.045,514.220$ & $063757.3-260000$ & 7.1 & 0.3 & $-7.30 \pm 0.07$ & $0.41 \pm 0.11$ & 0.12 \\
\hline E490-017-3-2509 & $657.358,639.546$ & $063757.7-255930$ & 8.4 & 0.2 & $-5.69 \pm 0.08$ & $1.10 \pm 0.11$ & 0.75 \\
\hline
\end{tabular}


M. E. Sharina et al.: HST imaging of globular cluster candidates in nearby LSB dwarf galaxies, Online Material p 4

Table 2. continued.

\begin{tabular}{|c|c|c|c|c|c|c|c|}
\hline GCC & $X, Y$ & RA (J2000) Dec & $r_{\mathrm{h}}$ & $e$ & $M_{V, 0}$ & $(V-I)_{0}$ & $d_{\text {proj }}$ \\
\hline KK065-3-1095 & $290.890,434.702$ & $074229.4+163429$ & 11.5 & 0.1 & $-6.75 \pm 0.08$ & $1.41 \pm 0.11$ & 0.33 \\
\hline U4115-2-1042 & $190.123,708.172$ & $075704.9+142225$ & 11.9 & 0.3 & $-6.00 \pm 0.08$ & $1.03 \pm 0.12$ & 1.72 \\
\hline $\mathrm{U} 4115-3-784$ & $614.000,125.730$ & $075703.8+142243$ & 9.4 & 0.1 & $-7.53 \pm 0.07$ & $0.93 \pm 0.11$ & 1.19 \\
\hline U4115-4-1477 & $607.416,740.185$ & $075704.1+142458$ & 8.0 & 0.0 & $-5.37 \pm 0.10$ & $0.91 \pm 0.16$ & 2.63 \\
\hline UA438-3-2004 & $299.313,413.097$ & $232628.3-322306$ & 3.7 & 0.2 & $-8.67 \pm 0.07$ & $0.96 \pm 0.10$ & 0.16 \\
\hline UA438-3-3325 & $733.228,621.063$ & $232626.7-322349$ & 3.7 & 0.1 & $-5.96 \pm 0.07$ & $1.02 \pm 0.10$ & 0.41 \\
\hline U3755-2-652 & $378.719,209.939$ & $071350.1+103215$ & 5.5 & 0.1 & $-8.22 \pm 0.07$ & $0.98 \pm 0.10$ & 1.84 \\
\hline U3755-2-675 & $107.413,234.823$ & $071350.4+103148$ & 8.1 & 0.1 & $-5.75 \pm 0.09$ & $1.19 \pm 0.12$ & 1.21 \\
\hline U3755-2-863 & $61.125,358.956$ & $071351.2+103144$ & 5.2 & 0.1 & $-6.93 \pm 0.08$ & $1.03 \pm 0.11$ & 0.96 \\
\hline U3755-3-727 & $494.971,57.246$ & $071352.1+103143$ & 9.5 & 0.1 & $-7.21 \pm 0.10$ & $0.60 \pm 0.15$ & 0.85 \\
\hline U3755-3-739 & $467.346,64.840$ & $071351.9+103142$ & 5.7 & 0.2 & $-8.67 \pm 0.07$ & $0.85 \pm 0.10$ & 0.83 \\
\hline U3755-3-754 & $380.674,72.749$ & $071351.3+103141$ & 8.1 & 0.0 & $-6.47 \pm 0.09$ & $0.56 \pm 0.14$ & 0.82 \\
\hline U3755-3-768 & $404.724,81.923$ & $071351.5+103140$ & 5.7 & 0.2 & $-5.42 \pm 0.10$ & $1.05 \pm 0.17$ & 0.79 \\
\hline U3755-3-914 & $379.331,139.121$ & $071351.3+103134$ & 9.5 & 0.0 & $-7.60 \pm 0.08$ & $0.64 \pm 0.11$ & 0.65 \\
\hline U3755-3-974 & $434.698,161.008$ & $071351.7+103132$ & 7.0 & 0.1 & $-5.70 \pm 0.01$ & $0.53 \pm 0.16$ & 0.58 \\
\hline U3755-3-1045 & $390.476,186.888$ & $071351.4+103129$ & 8.3 & 0.3 & $-6.01 \pm 0.10$ & $0.45 \pm 0.16$ & 0.53 \\
\hline U3755-3-1182 & $393.821,228.672$ & $071351.5+103125$ & 7.9 & 0.2 & $-8.54 \pm 0.07$ & $0.56 \pm 0.10$ & 0.43 \\
\hline U3755-3-1256 & $483.184,249.602$ & $071352.1+103124$ & 8.6 & 0.0 & $-7.77 \pm 0.07$ & $0.97 \pm 0.10$ & 0.37 \\
\hline U3755-3-1257 & $511.799,250.293$ & $071352.3+103124$ & 6.2 & 0.0 & $-8.71 \pm 0.07$ & $0.97 \pm 0.10$ & 0.40 \\
\hline U3755-3-1364 & $354.699,277.022$ & $071351.2+103120$ & 6.0 & 0.1 & $-7.00 \pm 0.08$ & $0.49 \pm 0.12$ & 0.36 \\
\hline U3755-3-1611 & $318.852,334.848$ & $071351.0+103114$ & 7.5 & 0.0 & $-7.48 \pm 0.08$ & $0.83 \pm 0.11$ & 0.34 \\
\hline U3755-3-1616 & $615.652,335.551$ & $071353.0+103116$ & 7.5 & 0.2 & $-6.40 \pm 0.09$ & $0.69 \pm 0.12$ & 0.46 \\
\hline U3755-3-1732 & $195.199,365.183$ & $071350.2+103111$ & 10.0 & 0.1 & $-6.09 \pm 0.08$ & $1.01 \pm 0.11$ & 0.62 \\
\hline U3755-3-1737 & $466.051,366.036$ & $071352.0+103112$ & 8.3 & 0.2 & $-6.71 \pm 0.08$ & $1.05 \pm 0.11$ & 0.09 \\
\hline U3755-3-1963 & $453.046,423.153$ & $071351.9+103106$ & 9.1 & 0.1 & $-6.46 \pm 0.09$ & $0.56 \pm 0.14$ & 0.09 \\
\hline U3755-3-2027 & $369.997,444.823$ & $071351.4+103104$ & 6.3 & 0.2 & $-5.80 \pm 0.10$ & $0.60 \pm 0.15$ & 0.22 \\
\hline U3755-3-2123 & $530.273,487.378$ & $071352.5+103100$ & 6.5 & 0.1 & $-7.76 \pm 0.07$ & $0.42 \pm 0.11$ & 0.33 \\
\hline U3755-3-2168 & $363.214,511.255$ & $071351.4+103057$ & 8.7 & 0.2 & $-7.63 \pm 0.07$ & $1.00 \pm 0.10$ & 0.36 \\
\hline U3755-3-2204 & $496.118,528.265$ & $071352.3+103056$ & 6.6 & 0.1 & $-6.07 \pm 0.09$ & $0.82 \pm 0.13$ & 0.37 \\
\hline U3755-3-2334 & $637.259,611.302$ & $071353.3+103049$ & 6.7 & 0.1 & $-6.90 \pm 0.07$ & $0.51 \pm 0.11$ & 0.74 \\
\hline U3755-3-2363 & $471.076,639.739$ & $071352.2+103045$ & 8.3 & 0.0 & $-7.75 \pm 0.07$ & $0.49 \pm 0.10$ & 0.63 \\
\hline U3755-3-2368 & $526.825,646.637$ & $071352.5+103045$ & 7.0 & 0.3 & $-7.44 \pm 0.07$ & $0.53 \pm 0.11$ & 0.68 \\
\hline U3755-3-2398 & $555.514,670.612$ & $071352.7+103043$ & 8.5 & 0.3 & $-6.17 \pm 0.09$ & $1.13 \pm 0.13$ & 0.76 \\
\hline $\mathrm{U} 3755-3-2401$ & $392.910,676.091$ & $071351.6+103041$ & 12.0 & 0.0 & $-7.54 \pm 0.07$ & $0.97 \pm 0.10$ & 0.73 \\
\hline U3755-3-2403 & $231.105,677.272$ & $071350.5+103040$ & 6.8 & 0.0 & $-6.79 \pm 0.07$ & $0.92 \pm 0.10$ & 0.89 \\
\hline U3755-3-2408 & $496.303,682.668$ & $071352.3+103041$ & 8.6 & 0.0 & $-6.89 \pm 0.08$ & $0.43 \pm 0.11$ & 0.75 \\
\hline U3755-3-2459 & $457.060,743.123$ & $071352.1+103035$ & 8.3 & 0.0 & $-7.98 \pm 0.07$ & $0.57 \pm 0.10$ & 0.89 \\
\hline U3755-4-566 & $188.706,59.303$ & $071348.9+103127$ & 8.6 & 0.3 & $-6.25 \pm 0.09$ & $0.87 \pm 0.14$ & 1.31 \\
\hline
\end{tabular}


M. E. Sharina et al.: HST imaging of globular cluster candidates in nearby LSB dwarf galaxies, Online Material p 5

Table 3. The King law approximation parameters for globular cluster candidates in nearby LSB dwarf galaxies. The table contains the following columns: identifier of each cluster (as in Table 2), reddening corrected $V$-band central surface brightness in mag/arcsec ${ }^{2}$ and corresponding error, reddening corrected $I$-band central surface brightness in mag/arcsec ${ }^{2}$ and corresponding error, King core radius $r_{\mathrm{c}}$ and corresponding error, King tidal radius $r_{\mathrm{t}}$ and corresponding error, and the King concentration parameter $c=r_{\mathrm{t}} / r_{\mathrm{c}}$.

\begin{tabular}{|c|c|c|c|c|c|}
\hline GCC & $\mu_{V, 0}$ & $\mu_{I, 0}$ & $r_{\mathrm{c}}$ & $r_{\mathrm{t}}$ & $c$ \\
\hline DDO53-3-1120 & $20.92 \pm 0.08$ & $19.67 \pm 0.01$ & $4.16 \pm 0.09$ & $21.3 \pm 1.0$ & 5.2 \\
\hline BK $3 N-2-863^{*}$ & $20.95 \pm 0.13$ & $20.70 \pm 0.03$ & $2.38 \pm 0.38$ & $26.4 \pm 4.5$ & 11.1 \\
\hline KDG73-2-378* & $21.33 \pm 0.05$ & $20.09 \pm 0.03$ & $3.47 \pm 0.21$ & 124.: & 36.0 \\
\hline KK77-4-939 & $20.64 \pm 0.68$ & $20.44 \pm 0.07$ & $2.26 \pm 0.22$ & $17.1 \pm 3.4$ & 7.6 \\
\hline KK $77-4-1162$ & $21.03 \pm 0.04$ & $20.35 \pm 0.05$ & $2.78 \pm 0.18$ & $18.0 \pm 2.4$ & 6.5 \\
\hline KK77-4-1165 & $21.42 \pm 0.05$ & $20.46 \pm 0.17$ & $3.19 \pm 0.22$ & $15.9 \pm 0.7$ & 5.0 \\
\hline KDG61-3-1325 & $18.45 \pm 0.03$ & $17.59 \pm 0.05$ & $1.88 \pm 0.06$ & $33.8 \pm 2.7$ & 18.0 \\
\hline KDG63-3-1168 & $18.93 \pm 0.04$ & $18.14 \pm 0.03$ & $1.90 \pm 0.06$ & $53.4 \pm 11.2$ & 28.1 \\
\hline DDO78-1-167 & $18.69 \pm 0.11$ & $17.75 \pm 0.03$ & $3.53 \pm 0.14$ & $46.7 \pm 6.8$ & 13.2 \\
\hline DDO78-3-1082 & $17.93 \pm 0.04$ & $16.98 \pm 0.02$ & $2.89 \pm 0.06$ & $34.6 \pm 2.1$ & 12.0 \\
\hline BK6N-2-524 & $20.40 \pm 0.13$ & $20.04 \pm 0.05$ & $1.54 \pm 0.15$ & $90.2 \pm 56.7$ & 58.7 \\
\hline BK6N-4-789 & $20.47 \pm 0.11$ & $19.66 \pm 0.01$ & $1.91 \pm 0.18$ & $68.0 \pm 8.1$ & 34.9 \\
\hline Garland-1-728 & $16.76 \pm 0.04$ & $15.90 \pm 0.08$ & $0.69 \pm 0.00$ & $42.3 \pm 14.6$ & 61.0 \\
\hline HoIX-3-866 & $17.88 \pm 0.07$ & $18.14 \pm 0.11$ & $1.51 \pm 0.06$ & $42.3 \pm 7.1$ & 28.0 \\
\hline HoIX-3-1168 & $19.69 \pm 0.07$ & $19.71 \pm 0.42$ & $2.00 \pm 0.15$ & $12.3 \pm 1.1$ & 6.2 \\
\hline HoIX-3-1322 & $20.02 \pm 0.01$ & $20.37 \pm 0.08$ & $2.34 \pm 0.00$ & $18.0 \pm 0.5$ & 7.7 \\
\hline HoIX-3-1565 & $19.34 \pm 0.31$ & $19.10 \pm 0.64$ & $1.06 \pm 0.44$ & $16.8 \pm 7.4$ & 13.5 \\
\hline HoIX-3-1664 & $19.28 \pm 0.11$ & $19.17 \pm 0.39$ & $1.18 \pm 0.09$ & $116 . \pm 87.2$ & 98.0 \\
\hline HoIX-3-1932 & $20.06 \pm 0.03$ & $19.60 \pm 0.07$ & $3.50 \pm 0.15$ & $23.0 \pm 2.6$ & 6.6 \\
\hline HoIX-3-2116 & $18.53 \pm 0.19$ & $18.65 \pm 0.06$ & $2.18 \pm 0.14$ & $21.4 \pm 3.0$ & 8.7 \\
\hline HoIX-3-2129 & $20.42 \pm 0.17$ & $20.30 \pm 0.21$ & $2.09 \pm 0.45$ & 975.: & 466.1 \\
\hline HoIX-3-2158 & $19.41 \pm 0.04$ & $19.44 \pm 0.07$ & $3.69 \pm 0.28$ & $11.8 \pm 0.9$ & 3.2 \\
\hline HoIX $-3-2373$ & $21.11 \pm 0.03$ & $19.70 \pm 0.06$ & $3.17 \pm 0.31$ & $22.3 \pm 2.6$ & 7.1 \\
\hline HoIX-3-2376 & $19.60 \pm 0.30$ & $20.34 \pm 0.16$ & $1.46 \pm 0.46$ & 76.3: & 52.4 \\
\hline HoIX-3-2409 & $18.31 \pm 0.12$ & $17.91 \pm 0.03$ & $1.91 \pm 0.06$ & $14.4 \pm 0.5$ & 7.5 \\
\hline HoIX-4-1038 & $17.06 \pm 0.10$ & $16.81 \pm 0.11$ & $2.08 \pm 0.19$ & $27.1 \pm 3.9$ & 13.1 \\
\hline HoIX-4-1085 & $20.24 \pm 0.27$ & $20.50 \pm 0.40$ & $1.40 \pm 0.29$ & $18.3 \pm 4.8$ & 13.2 \\
\hline E540-030-4-1183* & $21.13 \pm 0.02$ & $20.15 \pm 0.03$ & $2.92 \pm 0.09$ & $28.3 \pm 2.9$ & 9.7 \\
\hline E294-010-3-1104 & $20.96 \pm 0.05$ & $19.52 \pm 0.06$ & $3.64 \pm 0.50$ & $17.0 \pm 3.3$ & 4.7 \\
\hline KK027-4-721 & $20.39 \pm 0.09$ & $19.55 \pm 0.03$ & $3.33 \pm 0.11$ & $41.1 \pm 4.9$ & 12.3 \\
\hline Scu22-2-879 & $22.08 \pm 0.04$ & $20.77 \pm 0.05$ & $6.06 \pm 0.62$ & $51.3 \pm 16.9$ & 8.5 \\
\hline Scu22-2-100* & $20.72 \pm 0.04$ & $20.29 \pm 0.03$ & $3.44 \pm 0.20$ & $22.2 \pm 2.2$ & 6.5 \\
\hline Scu22-4-106* & $20.12 \pm 0.10$ & $19.44 \pm 0.07$ & $1.91 \pm 0.18$ & $33.4 \pm 7.0$ & 17.5 \\
\hline DDO113-2-579* & $21.26 \pm 0.05$ & $19.53 \pm 0.03$ & $2.73 \pm 0.14$ & $14.1 \pm 1.3$ & 5.2 \\
\hline DDO113-4-690 & $21.32 \pm 0.02$ & $20.18 \pm 0.07$ & $3.74 \pm 0.19$ & $19.4 \pm 2.4$ & 5.2 \\
\hline U7605-3-1503 & $21.49 \pm 0.07$ & $20.48 \pm 0.05$ & $6.16 \pm 0.71$ & $80.3 \pm 79.7$ & 13.1 \\
\hline KK109-3-1200* & $19.94 \pm 0.17$ & $19.40 \pm 0.20$ & $1.70 \pm 0.22$ & $19.2 \pm 3.0$ & 11.3 \\
\hline U7298-3-1280 & $20.56 \pm 0.16$ & $19.88 \pm 0.45$ & $2.42 \pm 0.45$ & $16.1 \pm 4.6$ & 6.7 \\
\hline U8308-2-1198 & $21.61 \pm 0.03$ & $20.17 \pm 0.06$ & $2.78 \pm 0.20$ & $27.4 \pm 3.7$ & 9.9 \\
\hline U8308-3-2040 & $20.62 \pm 0.08$ & $19.07 \pm 0.03$ & $3.25 \pm 0.17$ & $34.2 \pm 15.2$ & 10.5 \\
\hline U8308-4-893* & $20.03 \pm 0.02$ & $18.54 \pm 0.06$ & $1.91 \pm 0.00$ & $66.9 \pm 5.8$ & 35.0 \\
\hline U8308-4-971* & $21.83 \pm 0.06$ & $20.64 \pm 0.02$ & $3.68 \pm 0.30$ & 132. \pm 50.0 & 36.2 \\
\hline KK211-3-917 & $19.55 \pm 0.04$ & $18.98 \pm 0.03$ & $2.54 \pm 0.12$ & $51.9 \pm 13.9$ & 20.5 \\
\hline KK211-3-149 & $18.99 \pm 0.03$ & $18.18 \pm 0.05$ & $2.98 \pm 0.12$ & $113 . \pm 51.9$ & 38.1 \\
\hline KK221-2-608 & $18.03 \pm 0.03$ & $17.50 \pm 0.08$ & $1.78 \pm 0.06$ & $49.4 \pm 3.9$ & 27.8 \\
\hline KK221-2-883 & $20.04 \pm 0.03$ & $19.30 \pm 0.04$ & $3.43 \pm 0.17$ & $71.4 \pm 28.8$ & 20.8 \\
\hline KK221-2-966 & $16.57 \pm 0.04$ & $15.90 \pm 0.06$ & $2.26 \pm 0.11$ & $46.0 \pm 6.2$ & 20.4 \\
\hline KK221-2-1090 & $19.17 \pm 0.04$ & $18.58 \pm 0.02$ & $3.57 \pm 0.09$ & $30.6 \pm 3.3$ & 8.6 \\
\hline KK221-3-1062 & $21.77 \pm 0.06$ & $21.00 \pm 0.06$ & $5.85 \pm 0.85$ & $43.1 \pm 21.5$ & 7.4 \\
\hline KK200-3-1696 & $21.48 \pm 0.06$ & $20.87 \pm 0.04$ & $4.25 \pm 0.42$ & $38.0 \pm 13.2$ & 9.0 \\
\hline KK84-2-785 & $20.25 \pm 0.12$ & $19.69 \pm 0.13$ & $4.54 \pm 0.50$ & $39.9 \pm 5.3$ & 8.8 \\
\hline KK84-2-974 & $21.48 \pm 0.10$ & $21.08 \pm 0.04$ & $5.31 \pm 0.61$ & $38.5 \pm 3.9$ & 7.3 \\
\hline KK $84-3-705$ & $21.41 \pm 0.33$ & $20.03 \pm 0.10$ & $10.6 \pm 1.86$ & $36.8 \pm 14.1$ & 3.4 \\
\hline KK $84-3-830$ & $18.19 \pm 0.03$ & $17.16 \pm 0.10$ & $3.19 \pm 0.18$ & $90.4 \pm 4.0$ & 28.4 \\
\hline KK84-3-917 & $20.56 \pm 0.10$ & $19.62 \pm 0.17$ & $4.84 \pm 0.43$ & $40.9 \pm 4.7$ & 8.5 \\
\hline KK84-4-666 & $19.32 \pm 0.07$ & $18.44 \pm 0.03$ & $3.81 \pm 0.18$ & $45.3 \pm 1.8$ & 11.9 \\
\hline KK84-4-789 & $21.59 \pm 0.14$ & $20.94 \pm 0.10$ & $6.85 \pm 1.56$ & $57.7 \pm 34.1$ & 8.4 \\
\hline KK84-4-967 & $21.50 \pm 0.05$ & $20.61 \pm 0.14$ & $9.90 \pm 1.12$ & $22.2 \pm 1.7$ & 2.3 \\
\hline U9240-3-4557 & $18.37 \pm 0.01$ & $17.64 \pm 0.10$ & $1.81 \pm 0.00$ & $20.2 \pm 0.8$ & 11.2 \\
\hline KK112-3-976 & $21.03 \pm 0.03$ & $20.28 \pm 0.05$ & $3.37 \pm 0.12$ & $40.6 \pm 5.3$ & 12.1 \\
\hline KK112-4-742 & $21.40 \pm 0.06$ & $20.76 \pm 0.05$ & $4.64 \pm 0.41$ & $68.8 \pm 38.5$ & 14.8 \\
\hline KK112-4-792 & $21.26 \pm 0.03$ & $19.99 \pm 0.02$ & $6.20 \pm 0.20$ & $41.9 \pm 3.6$ & 6.8 \\
\hline E490-017-3-1769 & $19.49 \pm 0.03$ & $19.33 \pm 0.10$ & $3.16 \pm 0.15$ & $38.6 \pm 6.9$ & 12.2 \\
\hline E490-017-3-1861 & $18.86 \pm 0.08$ & $18.24 \pm 0.04$ & $2.76 \pm 0.12$ & $28.8 \pm 3.8$ & 10.5 \\
\hline E490-017-3-1956 & $20.85 \pm 0.28$ & $20.59 \pm 0.01$ & $2.74 \pm 0.09$ & $22.8 \pm 26.9$ & 8.3 \\
\hline E490-017-3-2035 & $19.23 \pm 0.03$ & $18.80 \pm 0.06$ & $3.27 \pm 0.18$ & $38.5 \pm 15.2$ & 11.8 \\
\hline E490-017-3-2509 & $21.00 \pm 0.01$ & $20.38 \pm 0.04$ & $4.79 \pm 0.09$ & $16.5 \pm 0.4$ & 3.5 \\
\hline
\end{tabular}


M. E. Sharina et al.: HST imaging of globular cluster candidates in nearby LSB dwarf galaxies, Online Material p 6

Table 3. continued.

\begin{tabular}{|c|c|c|c|c|c|}
\hline GCC & $\mu_{V 0}$ & $\mu_{I 0}$ & $r_{\mathrm{c}}$ & $r_{\mathrm{t}}$ & $c$ \\
\hline КK065-3-1095 & $20.96 \pm 0.03$ & $19.50 \pm 0.01$ & $5.28 \pm 0.12$ & $55.3 \pm 6.0$ & 10.5 \\
\hline $\mathrm{U} 4115-2-1042$ & $21.74 \pm 0.08$ & $21.07 \pm 0.07$ & $4.89 \pm 0.71$ & $293 .:$ & 59.9 \\
\hline U4115-3-784 & $19.82 \pm 0.14$ & $19.21 \pm 0.09$ & $3.11 \pm 0.48$ & $64.6 \pm 38.7$ & 20.8 \\
\hline U4115-4-1477 & $21.74 \pm 0.36$ & $20.96 \pm 0.05$ & $4.23 \pm 0.38$ & $25.7 \pm 5.1$ & 6.1 \\
\hline UA438-3-2004 & $17.05 \pm 0.01$ & $16.10 \pm 0.04$ & $1.73 \pm 0.04$ & $33.3 \pm 2.6$ & 19.3 \\
\hline UA438-3-3325 & $19.42 \pm 0.08$ & $18.62 \pm 0.03$ & $1.67 \pm 0.06$ & $31.2 \pm 19.8$ & 18.7 \\
\hline U3755-2-652 & $17.78 \pm 0.06$ & $17.19 \pm 0.03$ & $1.78 \pm 0.27$ & $33.5 \pm 7.7$ & 18.9 \\
\hline U3755-2-675 & $21.20 \pm 0.05$ & $20.24 \pm 0.34$ & $4.36 \pm 0.38$ & $18.6 \pm 1.9$ & 4.3 \\
\hline U3755-2-863 & $19.11 \pm 0.15$ & $18.92 \pm 0.02$ & $1.77 \pm 0.20$ & $42.6 \pm 2.8$ & 24.1 \\
\hline U3755-3-727 & $20.19 \pm 0.08$ & $19.82 \pm 0.03$ & $4.04 \pm 0.20$ & $141 . \pm 97.5$ & 35.1 \\
\hline U3755-3-739 & $18.00 \pm 0.09$ & $17.37 \pm 0.03$ & $2.30 \pm 0.09$ & $67.8 \pm 7.6$ & 29.5 \\
\hline U3755-3-754 & $20.34 \pm 0.05$ & $20.20 \pm 0.09$ & $3.93 \pm 0.51$ & $25.0 \pm 6.1$ & 6.4 \\
\hline U3755-3-768 & $20.98 \pm 0.21$ & $21.08 \pm 0.25$ & $2.25 \pm 0.41$ & $38.0 \pm 15.7$ & 17.0 \\
\hline U3755-3-914 & $19.84 \pm 0.04$ & $20.06 \pm 0.03$ & $3.43 \pm 0.18$ & $94.1 \pm 46.2$ & 27.5 \\
\hline U3755-3-974 & $21.22 \pm 0.04$ & $20.82 \pm 0.03$ & $3.47 \pm 0.20$ & $15.7 \pm 5.5$ & 4.5 \\
\hline U3755-3-1045 & $21.30 \pm 0.08$ & $21.02 \pm 0.09$ & $5.63 \pm 1.41$ & $17.5 \pm 4.8$ & 3.1 \\
\hline U3755-3-1182 & $18.39 \pm 0.02$ & $18.06 \pm 0.02$ & $3.54 \pm 0.09$ & $33.1 \pm 1.9$ & 9.4 \\
\hline U3755-3-1256 & $19.49 \pm 0.05$ & $18.82 \pm 0.01$ & $4.13 \pm 0.09$ & $36.6 \pm 1.5$ & 8.9 \\
\hline U3755-3-1257 & $17.74 \pm 0.03$ & $17.24 \pm 0.03$ & $2.15 \pm 0.09$ & $35.4 \pm 1.8$ & 16.5 \\
\hline U3755-3-1364 & $19.49 \pm 0.22$ & $19.52 \pm 0.08$ & $2.73 \pm 0.27$ & 283.: & 103.9 \\
\hline U3755-3-1611 & $19.29 \pm 0.05$ & $18.82 \pm 0.06$ & $2.70 \pm 0.12$ & $38.3 \pm 4.9$ & 14.2 \\
\hline U3755-3-1616 & $20.83 \pm 0.12$ & $20.39 \pm 0.07$ & $3.12 \pm 0.48$ & $60.7 \pm 32.7$ & 19.5 \\
\hline U3755-3-1732 & $21.04 \pm 0.08$ & $20.37 \pm 0.08$ & $3.89 \pm 0.50$ & $60.9 \pm 48.9$ & 15.7 \\
\hline U3755-3-1737 & $20.17 \pm 0.04$ & $19.95 \pm 0.05$ & $3.02 \pm 0.15$ & $34.6 \pm 4.5$ & 11.5 \\
\hline U3755-3-1963 & $20.91 \pm 0.12$ & $20.70 \pm 0.10$ & $4.50 \pm 1.03$ & 90.4: & 20.1 \\
\hline U3755-3-2027 & $20.85 \pm 0.04$ & $20.98 \pm 0.05$ & $3.53 \pm 0.20$ & $16.6 \pm 1.0$ & 4.7 \\
\hline U3755-3-2123 & $19.22 \pm 0.05$ & $19.18 \pm 0.11$ & $2.62 \pm 0.12$ & 344.: & 131.4 \\
\hline U3755-3-2168 & $19.54 \pm 0.03$ & $18.93 \pm 0.03$ & $4.18 \pm 0.18$ & $27.1 \pm 2.0$ & 6.5 \\
\hline U3755-3-2204 & $20.81 \pm 0.15$ & $20.27 \pm 0.04$ & $2.98 \pm 0.12$ & $19.8 \pm 6.4$ & 6.7 \\
\hline U3755-3-2334 & $19.90 \pm 0.05$ & $19.57 \pm 0.05$ & $3.34 \pm 0.22$ & $20.1 \pm 2.0$ & 6.0 \\
\hline U3755-3-2363 & $19.05 \pm 0.08$ & $18.86 \pm 0.07$ & $3.00 \pm 0.27$ & $39.7 \pm 9.4$ & 13.3 \\
\hline U3755-3-2368 & $19.82 \pm 0.02$ & $19.59 \pm 0.01$ & $3.35 \pm 0.09$ & $33.4 \pm 2.0$ & 10.0 \\
\hline U3755-3-2398 & $21.39 \pm 0.03$ & $20.77 \pm 0.06$ & $7.04 \pm 0.48$ & $18.0 \pm 1.1$ & 2.6 \\
\hline U3755-3-2401 & $19.92 \pm 0.04$ & $19.63 \pm 0.02$ & $4.07 \pm 0.22$ & $42.0 \pm 3.0$ & 10.3 \\
\hline U3755-3-2403 & $20.00 \pm 0.06$ & $19.11 \pm 0.10$ & $3.20 \pm 0.22$ & $20.0 \pm 2.0$ & 6.3 \\
\hline U3755-3-2408 & $20.21 \pm 0.06$ & $20.12 \pm 0.05$ & $3.59 \pm 0.31$ & $66.8 \pm 36.7$ & 18.6 \\
\hline U3755-3-2459 & $18.65 \pm 0.04$ & $18.74 \pm 0.05$ & $2.62 \pm 0.09$ & $49.0 \pm 5.1$ & 18.7 \\
\hline U3755-4-566 & $21.23 \pm 0.03$ & $20.59 \pm 0.06$ & $4.87 \pm 0.33$ & $53.6 \pm 19.4$ & 11.0 \\
\hline
\end{tabular}

